\title{
Effect of Irrigation Regime and Natural Soil Conditioner on Crop Productivity in Sandy Soil
}

\author{
Wafaa, M.T. El-etr, E.M. Aly and T. A. Eid \\ Soils, Water and Environ. Res. Inst., Agric.Res. Center (ARC), \\ Giza, Egypt.
}

\begin{abstract}
A FIELD experiment was conducted in sandy soil in Ismailia Governorate, Egypt, during two successive winter and summer seasons cultivated with lupine and sesame, respectively. The aim of this study was to evaluate the effects of three different water regimes (i.e., 0.8, 1.0, and 1.2 accumulative pan evaporation) and applied different rates $\left(3,4.5\right.$, and 6 ton fed $\left.^{-1}\right)$ of bentonite as mineral soil conditioner on crop productivity of both lupine and sesame crops under drip irrigation system. Total content of mineral nutrients of both tested plants were taken in consideration. Results indicated that, the highest actual evapotranspiration (ETa) was recorded under 1.2 accumulative pan evaporation treatment, for both lupine and sesame cropping system, while the lowest one was recorded under 0.8 accumulative pan evaporation. Also, applied 6.0 ton $\mathrm{fed}^{-1}$ bentonite recorded the lowest values of ETa in both seasons, whereas the highest value was obtained under zero ton $\mathrm{fed}^{-1}$ bentonite for the same respective crops. In addition, under Ismailia condition it could be concluded that pan evaporation and Hargreaves equations are more suitable to use for scheduling irrigation for lupine and sesame crops compared with the other equations. On the other hand, lupine and sesame yields (straw and grain/or seeds) along with total content of macronutrients ( $\mathrm{N}, \mathrm{P}$ and $\mathrm{K}$ ) increased significantly under the irrigation treatment of 1.2 accumulative pan evaporation in presenc of 6.0 ton $\mathrm{fed}^{-1}$ bentonite mineral soil conditioner as compared to other treatments.
\end{abstract}

Keywords: Sandy soil, Irrigation regime, Accumulative pan evaporation, Soil conditioner, Bentonite, Lupine, Sesame.

Sandy soils widely exist in arid and semi-arid regions such as the east and west desert areas of Egypt. Increasing the productive lands is now considered one of the major targets of the agricultural policy, however, the productivity of sandy soils is mostly limited by several agronomic obstacles. Also, sandy soils usually have poor properties including low specific surface area, low water retention, low organic matter content, low fertility and high infiltration rate. These poor physical properties cause insufficient water use, especially in arid and semi arid regions. These adverse factors can be solved by several means such as different natural conditioners (AL-Omran et al., 2004). Bentonite is widely used to improve poor soils in gardening, particularly, in sandy soils where small amounts 
of bentonite have been shown to increase growth rates and yields of many plants (Abd El-Hady \& El-dardiry, 2012 and Ferguson, 2015).

It is reported that calcium bentonite stores and releases water much easier than other types of clays (Matloub, 1998). It is also reported that applied soil conditioners (bentonite + zeolite) in the rate of 1.5, 2 and 2.5 ton fed ${ }^{-1}$ resulted in the greatest improvement in soil characteristics, particularly, under the highest rate of conditioner doses (Hassan and Mahmoud, 2013). Moreover, growth parameters of the crops and seed yields were beneficially increased with increasing the rate of applied conditioner. On the other hand, Afifi (1986) added that the addition of clay deposits (bentonite) to the sandy soil increased the retention and availability of soil moisture as well as the increase of the cohesive force among their practical. Abou-Gabal et al. (1990) found that the addition of local Tafla or shale (43\% silt, $24 \%$ clay and $32 \%$ fine sand dominated by bentonite) to the sandy soil in Egypt improved soil texture and consequently soilwater plant relationships. EL-Sherif and EL-Hady (1986) revealed that mixing sandy soil with local bentonite improved soil mechanical, hydro physical and chemical properties and consequently increased water use efficiency.

Schwen and Loiskandl (2010) revealed that inorganic soil amendments (IA) increased the available water capacity and resulted in a significant reduction of scheduled irrigation events and also a reduction of annual irrigation water requirements and water loss due to drainage for both climatic data of Brussels, Belgium (humid) and Cordoba, Spain (arid). Recently, Shakoor et al. (2012) showed that, the amendment of sandy soils with bentonite is one of the good options to decrease infiltration rate and increase water use efficiency.

On the other hand, approximately 5 million acres of lupine species are cultivated worldwide and $60 \%$ are used for seed production, while $40 \%$ used for forage and green manure. White lupine is a promising annual legume crop for human consumption, green manure, cover crops and forage production (Jansen, 2006). Podsiadło and Kotlarz (2001) stated that application of overhead irrigation and mineral fertilization on white lupine cultivated on sandy soil resulted in increasing the yield of seeds. Furthermore, the efficiency of NPK increased on watered plots. El-Fahal (2007) showed that lupine yield was significantly affected by irrigation interval and sowing date. Yield increased consistently with frequent irrigation and earlier sowing. Watering every 14 and 21 days reduced seed yield by 33 and $53 \%$ compared to the 7 day irrigation interval. To maximize the yield of lupine, it is advised to irrigate the crop every 7 to 14 day interval. Dmowski (1986) stated that the yield of yellow lupine can be obtained with over $300 \mathrm{~mm}$ of precipitation, while the optimum precipitation for yellow lupine was between 300 to $350 \mathrm{~mm}$.

Also, sesame is the most conventional oilseed crop cultivated for its edible oil in the sub-continent. Sesame is known as the king of oil seeds due to the high oil content $(50 \%-60 \%)$ of its seed (Toan et al., 2010). Sesame oil is used in food (cooking and salad), medicine and soap manufacturing. Jouyban and Moosavi (2011) showed that the increase in irrigation interval from 6 to 18 days decreased Egypt. J. Soil. Sci. 56, No. 2 (2016) 
seed yield, plant height, stem diameter, auxiliary branch number per plant and capsule length by 44.5, 35.8, 64.3, 49 and 45.7\%, respectively. Sarhadi and Sharif (2014) added that irrigation frequency treatments had a significant effect on sesame yield, yield components, plant height and number of branches. Increasing time distance between irrigation frequencies will lead to decreasing the yield, yield components and plant growth so the maximum of these parameters was obtained from irrigation frequency after $90 \mathrm{~mm}$ evaporation from pan. Ucan et al. (2007) indicated that obtaining the highest yield in sesame (1737 $\mathrm{kg} \mathrm{ha}^{-1}$ ) occurred when the crop was irrigated at interval as narrow as possible up to 6 days interval and irrigated under drip irrigation. They also showed that total applied water was 971 and $1037 \mathrm{~mm}$ and evapotranspiration rate sesame was 995 and $1111 \mathrm{~mm}$ (sprinkler), 1102 and $1135 \mathrm{~mm}$ (drip) in 1998 and 1999, respectively. Sepaskhah and Andam (2001) stated that the ET value for sesame was found to be $915.6 \mathrm{~mm}$ under semi-arid conditions. Nadeem et al. (2015) concluded that growth and yield attributes particularly, seed yield of sesame was significantly $(\mathrm{P}<0.05)$ affected by all the levels of irrigation regimes.

The aim of this study was to evaluate the irrigation scheduling using pan evaporation method with different rates of bentonite application, as mineral soil conditioner, on yield and macronutrients total content of both lupine and sesame cropping system grown in sandy soil.

\section{Materials and Methods}

Field experiments were conducted in sandy soil at Agricultural Research Station Farm in Ismailia Governorate, Egypt. The institute farm is located at $30^{\circ}$ 35' 41.9" N latitude and $32^{\circ} 16^{\prime} 45.8^{\prime \prime}$ E longitude. Lupine (Lupinus polyphyllus, cv. Giza 2) was cultivated during two successive winter seasons (2012/2013) and (2013/2014) and sesame (Sesamum indicum L, cv. Shandaweel 3) during two successive summer seasons of 2013 and 2014. The effect of irrigation regime and different rates of bentonite as mineral soil conditioner on lupine and sesame crop yields along with some plant - water relationships were, also, evaluated. Some physical and chemical characteristics of the studied soil are presented in Tables 1 and 2.

TABLE 1. Particle size distribution and chemical characteristics of the studied soil.

\begin{tabular}{|c|c|c|c|c|c|c|c|c|c|c|c|c|c|c|c|}
\hline \multirow{4}{*}{$\begin{array}{c}\text { Soil } \\
\text { depth } \\
\text { cm }\end{array}$} & \multicolumn{15}{|c|}{ Soil characteristics } \\
\hline & \multicolumn{5}{|c|}{$\begin{array}{c}\text { Particle size distribution } \\
\%\end{array}$} & \multicolumn{10}{|c|}{ Chemical analysis } \\
\hline & \multirow{2}{*}{$\begin{array}{c}\text { Coars } \\
\text { e } \\
\text { Sand }\end{array}$} & \multirow{2}{*}{$\begin{array}{l}\text { Fine } \\
\text { sand }\end{array}$} & \multirow[t]{2}{*}{ Silt } & \multirow[t]{2}{*}{ Clay } & \multirow{2}{*}{ Texture } & \multirow{2}{*}{ SP } & \multirow{2}{*}{$\underset{\mathbf{d S m}^{-1}}{\mathbf{E C}}$} & \multicolumn{4}{|c|}{ Anions (meq $\mathbf{L}^{-1}$ ) } & \multicolumn{4}{|c|}{ Cations (meq $\mathbf{L}^{-1}$ ) } \\
\hline & & & & & & & & $\mathrm{CO}^{-2}$ & $\mathrm{HCO}^{-}$ & $\mathrm{Cl}^{-}$ & $\mathrm{SO}^{-2}$ & $\mathrm{Ca}^{+2}$ & $\mathrm{Mg}^{+2}$ & $\mathrm{Na}^{+}$ & $\overline{\mathbf{K}^{+}}$ \\
\hline $0-15$ & 68.00 & 25.75 & 3.82 & 2.43 & sandy & 22.7 & 3.71 & - & 11.8 & 21.5 & 1.50 & 11.07 & 5.88 & 14.5 & 3.35 \\
\hline $15-30$ & 72.32 & 23.07 & 3.11 & 1.50 & sandy & 20.0 & \begin{tabular}{|l|}
0.44 \\
\end{tabular} & - & 1.94 & 1.79 & 0.56 & 1.23 & 0.49 & 2.25 & 0.32 \\
\hline $30-45$ & 75.20 & 20.97 & 3.00 & 0.83 & sandy & 20.7 & \begin{tabular}{|l|}
0.22 \\
\end{tabular} & - & 1.47 & 0.89 & 0.52 & 0.62 & 0.25 & $\begin{array}{ll}1.57 \\
\end{array}$ & \begin{tabular}{|l|}
0.44 \\
\end{tabular} \\
\hline $45-60$ & 87.44 & 8.46 & 3.65 & 0.45 & sandy & 22.7 & 0.25 & - & 1.47 & 0.89 & 0.54 & 0.62 & 0.49 & 1.38 & 0.41 \\
\hline
\end{tabular}


TABLE 2. Soil bulk densities and moisture constants after added bentonite to the studied soil site.

\begin{tabular}{|c|c|c|c|c|c|c|c|}
\hline \multirow[t]{2}{*}{$\begin{array}{l}\text { Bentonite } \\
\text { rates }\end{array}$} & \multirow[t]{2}{*}{$\begin{array}{l}\text { Soil depth } \\
\quad(\mathrm{cm})\end{array}$} & \multirow[t]{2}{*}{$\begin{array}{l}\text { Bulk density } \\
\mathbf{c m}^{-3}\end{array}$} & \multicolumn{2}{|c|}{$\begin{array}{c}\text { Retained moisture at field } \\
\text { capacity, } \mathrm{v} / \mathrm{v}\end{array}$} & \multicolumn{2}{|c|}{$\begin{array}{c}\text { Retained moisture at } \\
\text { permanent wilting } \\
\text { point } v / v\end{array}$} & \multirow{2}{*}{$\begin{array}{c}\text { Available } \\
\text { moisture } \\
\text { mm/soil depth }\end{array}$} \\
\hline & & & $\%$ & 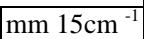 & $\%$ & $\mathrm{~mm}-15 \mathrm{~cm}^{-1}$ & \\
\hline \multirow{5}{*}{0 ton fed $^{-1}$} & $0-15$ & 1.58 & 12.17 & 18.25 & 2.13 & 3.20 & 15.05 \\
\hline & $15-30$ & 1.62 & 11.01 & 16.52 & 2.01 & 3.02 & 13.51 \\
\hline & $30-45$ & 1.64 & 10.17 & 15.25 & 2.53 & 3.80 & 11.46 \\
\hline & $45-60$ & 1.66 & 7.97 & 11.95 & 2.66 & 3.99 & 8.03 \\
\hline & & & & & & & 48.05 \\
\hline \multirow{5}{*}{3 ton $\mathrm{fed}^{-1}$} & $0-15$ & 1.57 & 13.21 & 19.82 & 2.18 & 3.27 & 16.55 \\
\hline & $15-30$ & 1.62 & 11.16 & 16.74 & 2.59 & 3.89 & 12.85 \\
\hline & $30-45$ & 1.64 & 10.17 & 15.25 & 2.44 & 3.66 & 11.95 \\
\hline & $45-60$ & 1.66 & 7.97 & 11.95 & 2.61 & 3.92 & 8.03 \\
\hline & & & & & & & 49.38 \\
\hline \multirow{5}{*}{4.5 ton $\mathrm{fed}^{-1}$} & $0-15$ & 1.55 & 13.65 & 20.48 & 2.2 & 3.3 & 17.18 \\
\hline & $15-30$ & 1.62 & 11.16 & 16.74 & 2.59 & 3.89 & 12.85 \\
\hline & $30-45$ & 1.64 & 10.17 & 15.25 & 2.44 & 3.66 & 11.95 \\
\hline & $45-60$ & 1.66 & 7.97 & 11.95 & 2.61 & 3.92 & 8.03 \\
\hline & & & & & & & 50.01 \\
\hline \multirow{5}{*}{6.0 ton $\mathrm{fed}^{-1}$} & $0-15$ & 1.52 & 15.2 & 22.8 & 2.26 & 3.39 & 19.41 \\
\hline & $15-30$ & 1.62 & 11.60 & 17.86 & 2.15 & 3.23 & 14.63 \\
\hline & $30-45$ & 1.64 & 10.17 & 15.25 & 2.44 & 3.66 & 11.95 \\
\hline & $45-60$ & 1.66 & 7.97 & 11.95 & 2.61 & 3.92 & 8.03 \\
\hline & & & & & & & 54.02 \\
\hline
\end{tabular}

\section{The meteorological data}

Air temperature $\left({ }^{\circ} \mathrm{C}\right)$ relative humidity $(\%)$, actual and possible sunshine (hour), solar and extraterrestrial radiation $\left(\mathrm{MJ} \mathrm{m}^{-2} \mathrm{day}^{-1}\right)$ and wind speed $\left(\mathrm{m} \mathrm{sec}^{-1}\right)$ at Ismailia Station had been daily recorded and their monthly mean values were calculated during the last ten years period (Table 3 ).

TABLE 3. Average of the meteorological data of Ismailia station during the last ten years period.

\begin{tabular}{|c|c|c|c|c|c|c|c|c|c|c|c|}
\hline \multirow[b]{2}{*}{ Month } & \multicolumn{11}{|c|}{ Parameters } \\
\hline & $\begin{array}{c}\mathbf{T}_{\max } \\
{ }^{\mathbf{0}} \mathbf{C} \\
\end{array}$ & $\begin{array}{c}\mathbf{T}_{\min } . \\
{ }^{\mathbf{0}} \mathbf{C}\end{array}$ & $\mathbf{T}_{\text {mean }}{ }^{\circ} \mathbf{C}$ & $\begin{array}{c}\mathbf{R H}_{\text {max. }} \\
\%\end{array}$ & $\mathbf{R H}_{\min } \%$ & $\begin{array}{c}\mathbf{R H}_{\text {mean }} \\
\%\end{array}$ & $\begin{array}{c}\mathrm{W} . \mathrm{S} \\
\mathrm{m} / \mathrm{sec}\end{array}$ & $\begin{array}{l}\text { ASD } \\
\text { hour }\end{array}$ & $\begin{array}{l}\text { PSD } \\
\text { hour }\end{array}$ & \begin{tabular}{|l|} 
STR (MJ \\
m $^{-2}$ day $\left.^{-1}\right)$ \\
\end{tabular} & \begin{tabular}{|l} 
ETR (MJ \\
m $^{-2}$ day $\left.^{-1}\right)$
\end{tabular} \\
\hline January & 19.2 & 8.1 & 13.65 & 87.2 & 21.5 & 54.35 & 1.7 & 6.89 & 10.4 & 11.7 & 20.9 \\
\hline February & 20.6 & 8.3 & 14.45 & 86.1 & 18.6 & 52.35 & 1.9 & 7.59 & 11.1 & 14.9 & 25.6 \\
\hline march & 23.8 & 10.7 & 17.3 & 86.3 & 22.5 & 54.40 & 2.0 & 8.46 & 12.0 & 18.5 & 31.3 \\
\hline April & 28.6 & 13.9 & 21.3 & 88.6 & 19.6 & 54.10 & 2.1 & 9.38 & 12.9 & 22.0 & 36.8 \\
\hline may & 32.8 & 18.0 & 25.4 & 88.2 & 21.0 & 54.60 & 2.0 & 10.3 & 13.6 & 24.7 & 40.0 \\
\hline June & 34.5 & 21.5 & 28.0 & 87.1 & 23.6 & 55.40 & 1.6 & 12.9 & 14.0 & 27.7 & 41.2 \\
\hline July & 36.4 & 23.1 & 29.8 & 91.4 & 27.7 & 59.60 & 1.6 & 12.7 & 13.9 & 27.1 & 40.6 \\
\hline August & 35.9 & 23.8 & 29.9 & 90.4 & 26.1 & 58.30 & 1.6 & 11.5 & 13.2 & 25.3 & 38.0 \\
\hline September & 33.1 & 21.7 & 27.4 & 87.7 & 30.0 & 58.70 & 1.8 & 10.6 & 12.4 & 22.0 & 33.3 \\
\hline October & 29.7 & 19.9 & 24.8 & 88.1 & 21.7 & 54.90 & 1.4 & 9.27 & 11.5 & 17.5 & 27.4 \\
\hline November & 26.3 & 16.0 & 21.2 & 88.3 & 22.5 & 55.40 & 1.4 & 7.88 & 10.6 & 13.3 & 22.0 \\
\hline December & 20.6 & 10.5 & 15.6 & 86.6 & 21.7 & 54.20 & 1.7 & 6.82 & 10. & 11.0 & 19.6 \\
\hline
\end{tabular}

Egypt. J. Soil. Sci. 56, No. 2 (2016) 
T: temperature, RH: relative humidity, WS: wind speed, ASD: actual sun shine duration, PSD: potential sun shine duration, STR: solar terrestrial radiation, ETR: extra terrestrial radiation. Irrigation system

The experiment was irrigated by a surface drip irrigation system. The tubes were spaced $0.6 \mathrm{~m}$ apart. The drip line tubing has $0.3 \mathrm{~m}$ spacing distances between emitters and a flow rate of $4.0 \mathrm{~L} \mathrm{~h}^{-1} \mathrm{~m}^{-1}$ at design operating pressure of $206 \mathrm{kPa}$.

\section{Experimental layout}

Lupine as a winter crop followed by sesame crop as a summer crop, both of them were sown in agricultural cycle. This rotation was repeated for two years. Lupine was sown on $25 / 11 / 2012$ and $27 / 11 / 2013$ for the first and second year, respectively, while the corresponding dates for sesame crop were sown on 5/6/2013 and 1/6/2014 for the first and second year, respectively. The experiment was arranged in split plot design with three replicates. The main plot was assigned as irrigation treatments while the sub plot treatment was bentonite rates. The main plots consisted of three irrigation treatments $(0.8,1.0$ and 1.2 pan evaporation coefficient). The subplots consisted of four rates of bentonite conditioner (3, 4.5 and 6 ton $\mathrm{fed}^{-1}$ ). All cultural practices were the same as recommended for the two crops except for different irrigation treatments used. Bentonite treatments were added before cultivation and mixed with soil surface $20 \mathrm{~cm}$. Mineral fertilizers were applied at the recommended doses for both crops. Superphosphate $\left(15 \% \mathrm{P}_{2} \mathrm{O}_{5}\right)$ at a rate of $20 \mathrm{~kg} \mathrm{fed}^{-1}$ added basically before sowing during soil preparation. Nitrogen added at rates of $15 \mathrm{~kg} \mathrm{fed}^{-1}$ and $150 \mathrm{~kg} \mathrm{fed}^{-1}$ forbothlupine and sesame, respectively, in three split equal doses after 15 , 30 and 60 days from sowing in the form of ammonium sulfate $(20 \% \mathrm{~N})$, while potassium added at the form potassium sulfate $\left(48 \% \mathrm{~K}_{2} \mathrm{O}\right)$ at a rate of $50 \mathrm{kgfed}^{-1}$ in two equal doses at sowing and 30 days from sowing for both lupine and sesame. Irrigation was practiced according to the daily records of the evaporations pan and the crops, i.e., lupine and sesame were irrigated when the water balance reached zero, theoretically. This means, when daily evaporation pan reading multiplied by evaporation coefficient equals total available soil moisture.

\section{The study characters}

Water relations

The consumptive use $(\mathrm{Cu})$ or actual evapotranspiration $\left(E T_{a}\right)$

The consumptive use $(\mathrm{Cu})$ of water estimated according to the equation given by Israelson and Hansen (1962) as follows:

$$
\mathrm{Cu}=\frac{\operatorname{Dad}\left(\mathrm{e}_{2}-\mathrm{e}_{1}\right)}{100}
$$

where:

$$
\begin{aligned}
& \mathrm{Cu}=\text { depth of irrigation application }[\mathrm{mm}], \\
& \mathrm{D}=\text { depth of irrigation }[\mathrm{mm}] \\
& \mathrm{Ad}=\text { soil bulk density }\left[\mathrm{g} / \mathrm{cm}^{3}\right], \\
& \mathrm{e}_{1}=\text { soil moisture content before irrigation, }[\mathrm{w} / \mathrm{w}], \\
& \mathrm{e}_{2}=\text { soil moisture content after irrigation, }[\mathrm{w} / \mathrm{w}] .
\end{aligned}
$$

Potential evapotranspiration $\left(E T_{o}\right)$ :

Potential evapotranspiration $\left(\mathrm{ET}_{\mathrm{o}}\right)$ was determined by five methods: 
Pan evaporation method: The pan evaporation is related to the reference evapotranspiration described by Doorenbos and Pruitt (1977) as follows:

$$
\mathrm{ET}_{\mathrm{o}}=\mathrm{K}_{\mathrm{p}} \times \mathrm{E}_{\mathrm{pan}}
$$

where: $\mathrm{ET}_{\mathrm{o}}$ reference evapotranspiration [mm/day],

$\mathrm{K}_{\mathrm{p}}$ Pan Coefficient [-],

$\mathrm{E}_{\mathrm{p}}$ Pan Evaporation [mm/day].

Penman-Monteith method (mm/day): The Penman Monteith daily $\left(\mathrm{PM}_{\mathrm{d}}\right)$ equation is as follows:

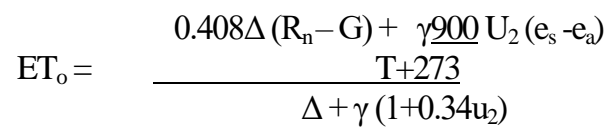

where $\mathrm{ET}_{\mathrm{o}}$ reference evapotranspiration $\left[\mathrm{mm} \mathrm{day}^{-1}\right]$,

$\mathrm{R}_{\mathrm{n}}$ net radiation at the crop surface $\left[\mathrm{MJm}^{-2}\right.$ day $\left.^{-1}\right]$

$\mathrm{G}$ soil heat flux density $\left[\mathrm{M} \mathrm{J} \mathrm{m}^{-2}\right.$ day $\left.^{-1}\right]$,

T mean daily air temperature at $2 \mathrm{~m}$ height $\left[{ }^{\circ} \mathrm{C}\right]$

$\mathrm{U}_{2}$ wind spaced at $2 \mathrm{~m}$ height $\left[\mathrm{ms}^{-1}\right]$,

$\mathrm{e}_{\mathrm{s}}$ saturation vapour pressure $\left[\mathrm{K} P_{\mathrm{a}}\right]$,

ea actual vapour pressure $[\mathrm{K} P \mathrm{a}$,

$\mathrm{e}_{\mathrm{s}}-\mathrm{e}_{\mathrm{a}}$ saturation vapour pressure deficit $\left[\mathrm{K} P_{\mathrm{a}}\right]$,

$\Delta$ slope vapour pressure curve $\left[\mathrm{K} P_{\mathrm{a}}{ }^{\mathrm{o}-1} \mathrm{C}\right]$,

$\gamma$ prychrometric constant $\left[\mathrm{K} P_{\mathrm{a}}{ }^{\mathrm{o}-1} \mathrm{C}\right]$.

Hargreaves and Samani (1985) reference evapotranspiration equation:

$$
\mathrm{ET}_{\mathrm{o}}=0.0023\left(\mathrm{~T}_{\text {mean }}+17.8\right)\left(\mathrm{T}_{\max }-\mathrm{T}_{\text {min }}\right)^{0.5} \mathrm{Ra}
$$

FAO modified Penman equation (1977):

$$
E_{\mathrm{o}}=c\left[w \cdot R_{n}+(1-w) \cdot f(u) \cdot\left(e_{a}-e_{d}\right)\right.
$$

where:

$e_{a}-e_{d}=$ vapour pressure deficit, i.e. the difference between saturation vapour $\mathrm{e}_{\mathrm{a}}$ at $\mathrm{T}_{\text {mean }}$ (in mbar) and actual vapour pressure $\left(\mathrm{e}_{\mathrm{d}}\right)$ where $\mathrm{e}_{\mathrm{d}}=\mathrm{e}_{\mathrm{a}} \mathrm{RH}_{\text {mean }} / 100, \mathrm{f}(\mathrm{u})=$ wind function $\mathrm{f}(\mathrm{u})=$ $0.27(1+\mathrm{u} / 100)$ with $\mathrm{u}$ in $\mathrm{km} /$ day measured at $2 \mathrm{~m}$ height.

$$
\mathrm{R}_{\mathrm{n}}=0.75 \mathrm{R}_{\mathrm{s}}-\mathrm{R}_{\mathrm{nl}}
$$

$R_{s}$ is incoming short wave radiation in mm/day either measured or obtained from $R_{s}=$ $(0.25+0.5 n / N) R_{a} \cdot R_{a}$ is extra-terrestrial radiation in mm/day, $n$ is the mean actual sunshine duration in hour/day and $\mathrm{N}$ is maximum possible sunshine duration hour/day. $\mathrm{R}_{\mathrm{nl}}$ is net long 
wave radiation in $\mathrm{mm} /$ day and is a function of temperature, $\mathrm{f}(\mathrm{t})$, actual vapour pressure, $\mathrm{f}\left(\mathrm{e}_{\mathrm{d}}\right)$ and sunshine duration, $\mathrm{f}(\mathrm{n} / \mathrm{N})$, or $\mathrm{R}_{\mathrm{nl}}=\mathrm{f}(\mathrm{t}) . \mathrm{f}\left(\mathrm{e}_{\mathrm{d}}\right) . \mathrm{f}(\mathrm{n} / \mathrm{N})$.

$\mathrm{W}=$ temperature and altitude dependent weighting factor.

$\mathrm{C}=$ adjustment factor for $\mathrm{U}$ day, ratio of $\mathrm{U}$ day $/ \mathrm{U}$ night, $\mathrm{RH}_{\max .}$ and $\mathrm{R}_{\mathrm{s}}$.

FAO modified Blaney-Criddle method, according to Cuenca and Amegee (1987)

where:

$$
\mathrm{ET}_{\mathrm{r}}=\{\mathrm{a}+\mathrm{b}[\mathrm{p}(0.46 \mathrm{~T}+8.13)]\}[1.0+0.1(\mathrm{E} / 100)]
$$

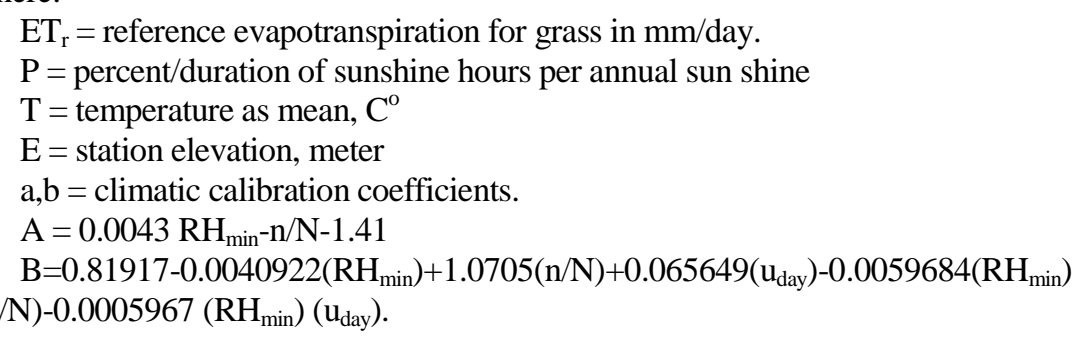

\section{Crop coefficient $\left(K_{c}\right)$}

The recommended values of Kc, according to Doorenbos and Kassam (1979) were used to estimate the $\mathrm{ET}_{\mathrm{o}}$ for the conditions of the area where the experiment was done. The formula is as follows:

$$
\mathrm{ET}_{\mathrm{c}}=\mathrm{K}_{\mathrm{c}} \times \mathrm{ET}_{\mathrm{o}}
$$

where: $\mathrm{K}_{\mathrm{c}}$ : Crop coefficient.

$\mathrm{ET}_{\mathrm{c}}$ : The measured (estimate) evapotranspition of a considered period ( $\mathrm{mmday}^{-1}$ ) $\mathrm{ET}_{\mathrm{o}}$ : reference evapotranspiration $\left(\right.$ mmday $\left.^{-1}\right)$ referring to the same period, calculated as average value of four formulae.

Water use efficiency

Water use efficiency (WUE) in $\mathrm{kg} / \mathrm{m}^{3}$ was calculated for the deferent treatments, using the following formulae of Vites (1965):

$$
\text { W.U.E }=\frac{\text { Seed yield in } \mathrm{kg} / \mathrm{fed}}{\text { Actual evapotranspiration in } \mathrm{m}^{3} \mathrm{fed}^{-1}}
$$

Dates of harvesting for lupine were 28/4/2013 and 30/4/2014 while, for sesame crop took place on 28/9/2013 and 24/9/2014, straw and grains for both lupine and sesame crops collected from each plot, oven dried at $70^{\circ} \mathrm{C}$ for $48 \mathrm{hr}$, and weighed up to a constant dry weight, ground and prepared for digestion according to Page et al. (1982). The digests were then exposed to the estimation of N, P, K (Cottenie et al., 1982).

\section{Statistical analysis}

All the data collected for the yield and its components and chemical composition were subjected to the statistical analysis according to Snedecor and Cochran (1967) and the mean values were compared by LSD at $5 \%$. 


\section{Results and Discussion}

Water relations of both lupine and sesame crops rotation:

Lupine actual evapotranspiration $\left(E T_{a}\right)$

Evapotranspiration (ET) is an important term in the water balance of a cropped area. Irrigation engineers need to know how much of the applied irrigation water is consumed by the crop; even calculate, or estimate, the remaining components of the water balance. On the other hand, agriculturists need to know the specific water requirements for a crop so that they can obtain a satisfactory yield. Reduction in ET due to plant-water stress, caused by water shortage, is associated with reduced plant yield, since both ET and photosynthesis are functions of stomata regulation.

Lupine periodical crop seasonal actual evapotranspiration as affected by irrigation regime and bentonite soil conditioner during the growing two winter seasons of $2012 / 2013$ and 2013/2014 is presented in Table 4. Results of first season indicated that seasonal actual evapotranspiration, $\mathrm{ET}_{\mathrm{a}}$, values were 437.69, 360.88 and 313.48 $\mathrm{mm}$ resulted from 1.2, 1.0 and 0.8 accumulative pan evaporation treatments, respectively. Whereas the corresponding values for the second season were 452.96, 361.77 and $297.65 \mathrm{~mm}$ for the same respective irrigation regimes. In general, results in both season indicated that, the highest $\mathrm{ET}_{\mathrm{a}}$ was recorded under 1.2 accumulative pan evaporation treatment, while the lowest one was recorded under 0.8 treatment. From these results, it could be concluded that the quantity of water use depends on frequent irrigation intervals. When soil was kept wet by short irrigation intervals, higher seasonal consumptive use was obtained. Similar results were obtained by ElFahal (2007).

With respect to the effect of bentonite soil conditioner rates application, data presented in Table 4 showed that average values of $\mathrm{ET}_{\mathrm{a}}$ in first season were 385.1, 374.5, 367.2 and $355.9 \mathrm{~mm}$ for adding 6.0, 4.5, 3.0 and zero ton $\mathrm{fed}^{-1}$ bentonite application, respectively, while in the second season, results indicated that similar values were $392.5,377.1,363.8$ and $349.6 \mathrm{~mm}$ for the same respective bentonite treatments. The lowest and highest values were recorded by adding 6.0 and zero ton $\mathrm{fed}^{-1}$, respectively. Due to the improvement in soil ability, a retain water and increased soil available water under coarse textured soils may be performed (Schwen and Loiskandl, 2010).

Finally, it can be concluded that the optimum pan evaporation coefficient (1.0) could be considered as the optimum irrigation treatment to obtain the highest seed yield of lupine crop ( 360.88 and $361.77 \mathrm{~mm}$ ) water consumptive use in both growing seasons, respectively and it could be used in scheduling irrigation for lupine crop.

\section{Sesame actual evapotranspiration $\left(E T_{a}\right)$}

Seasonal actual evapotranspiration as affected by irrigation regimes and bentonite condition during 2013 and 2014 are presented in Table 5. Results of the first season indicate that seasonal actual evapotranspiration $\left(\mathrm{ET}_{\mathrm{a}}\right)$ values were $784.75,722.38$ and 
EFFECT OF IRRIGATION REGIME AND NATURAL SOIL CONDITIONER 335

$633.55 \mathrm{~mm}$ resulted from 1.2, 1.0 and 0.8 accumulative pan evaporation treatments, respectively.

TABLE 4. Lupine daily, monthly and total actual evapotranspiration $\left(\mathbf{E T}_{\mathrm{a}, \mathrm{mm}}\right)$ as affected by different values of pan coefficient and bentonite conditioner rates.

\begin{tabular}{|c|c|c|c|c|c|c|c|c|c|c|c|c|c|c|c|}
\hline \multicolumn{16}{|c|}{ Lupine season 2012/2013 } \\
\hline \multicolumn{2}{|c|}{ months } & \multicolumn{2}{|c|}{ Nov." } & \multicolumn{2}{|c|}{ Dec. } & \multicolumn{2}{|r|}{ Jan. } & \multicolumn{2}{|c|}{ Feb. } & \multicolumn{2}{|c|}{ Mar. } & \multicolumn{2}{|c|}{ Apr. ${ }^{* *}$} & \multirow{2}{*}{\multicolumn{2}{|c|}{ Total }} \\
\hline \multirow{2}{*}{\begin{tabular}{|c|} 
Irrg. \\
Regime
\end{tabular}} & \multirow{2}{*}{$\begin{array}{c}\text { Bent. } \\
\text { ton / fed. }\end{array}$} & Daily & monthly & Daily & monthly & Daily & monthly & Daily & monthly & Daily & monthly & Daily & monthly & & \\
\hline & & $\mathrm{mm}$ & $\mathrm{mm}$ & $\mathrm{mm}$ & $\mathrm{mm}$ & $\mathrm{mm}$ & $\mathrm{mm}$ & $\mathrm{mm}$ & $\mathrm{mm}$ & $\mathrm{mm}$ & $\mathrm{mm}$ & $\mathrm{mm}$ & $\mathrm{mm}$ & $\mathrm{mm}$ & $\mathrm{m}^{3}$ fed. \\
\hline \multirow{4}{*}{$\begin{array}{l}1.2 \\
\text { Pan. }\end{array}$} & 6 & 3.21 & 19.27 & 1.76 & 54.41 & 2.35 & 72.74 & 2.56 & 71.73 & 4.68 & 145.1 & 2.05 & 55.25 & 418.5 & 1757.9 \\
\hline & 4.5 & 3.21 & 19.27 & 1.81 & 56.01 & 2.47 & 76.54 & 2.68 & 74.94 & 4.88 & 151.3 & 2.11 & 56.91 & 434.9 & 1826.8 \\
\hline & 3 & 3.21 & 19.27 & 1.84 & 57.02 & 2.52 & 78.25 & 2.73 & 76.55 & 4.93 & 152.7 & 2.16 & 58.34 & 442.1 & 1856.9 \\
\hline & 0 & 3.21 & 19.27 & 1.88 & 58.37 & 2.58 & 79.94 & 2.83 & 79.30 & 5.09 & 157.8 & 2.24 & 60.46 & 455.1 & 1911.4 \\
\hline \multicolumn{2}{|c|}{ Mean } & 3.21 & 19.27 & 1.82 & 56.45 & 2.48 & 76.87 & 2.70 & 75.63 & 4.89 & 151.73 & 2.14 & 57.74 & 437.65 & 51838.3 \\
\hline \multirow{4}{*}{$\begin{array}{l}1.0 \\
\text { Pan }\end{array}$} & 6 & 3.21 & 19.27 & 1.16 & 35.95 & 2.14 & 66.44 & 2.56 & 71.69 & 3.20 & 99.35 & 2.05 & 55.23 & 347.9 & 1461.3 \\
\hline & 4.5 & 3.21 & 19.27 & 1.18 & 36.71 & 2.19 & 67.87 & 2.65 & 74.27 & 3.29 & 102.1 & 2.10 & 56.60 & 356.9 & 1498.8 \\
\hline & 3 & 3.21 & 19.27 & 1.22 & 37.7 & 2.21 & 68.52 & 2.73 & 76.31 & 3.37 & 104.5 & 2.10 & 56.57 & 362.9 & 1524.1 \\
\hline & 0 & 3.21 & 19.27 & 1.23 & 38.20 & 2.30 & 71.34 & 2.83 & 79.15 & 3.48 & 107.8 & 2.23 & 60.15 & 376.3 & 1580.3 \\
\hline \multicolumn{2}{|c|}{ Mean } & \begin{tabular}{|l}
3.21 \\
\end{tabular} & 19.27 & 1.20 & 37.14 & 2.21 & 68.54 & 2.70 & 75.35 & 3.30 & 103.44 & 2.10 & 57.13 & 360.88 & \begin{tabular}{l|l} 
& 1516.1 \\
\end{tabular} \\
\hline \multirow{4}{*}{$\begin{array}{l}0.8 \\
\text { Pan }\end{array}$} & 6 & \begin{tabular}{|l|}
3.21 \\
\end{tabular} & 19.27 & 1.15 & 38.2 & 1.79 & 59.29 & 2.42 & 73.27 & 2.86 & 95.80 & 1.27 & 38.42 & 324.3 & 1361.9 \\
\hline & 4.5 & 3.21 & 19.27 & 1.18 & 37.69 & 1.85 & 58.26 & 2.52 & 71.55 & 2.95 & 94.34 & 1.28 & 37.36 & 318.5 & 1337.6 \\
\hline & 3 & 3.21 & 19.27 & 1.22 & 36.58 & 1.88 & 57.26 & 2.56 & 70.50 & 3.04 & 91.57 & 1.38 & 34.68 & 309.9 & 1301.4 \\
\hline & 0 & 3.21 & 19.27 & 1.23 & 35.73 & 1.91 & 55.59 & 2.62 & 67.78 & 3.09 & 88.63 & 1.42 & 34.29 & 301.3 & 1265.4 \\
\hline \multicolumn{2}{|c|}{ Mean } & 3.21 & 19.27 & 1.20 & 37.05 & 1.86 & 57.60 & 2.53 & 70.78 & 2.99 & 92.59 & 1.34 & 36.19 & 313.48 & \begin{tabular}{l|l}
8 & 1316.6
\end{tabular} \\
\hline \multirow{4}{*}{$\begin{array}{r}\text { Overall } \\
\text { Mean for } \\
\text { Bentonite }\end{array}$} & 6 & 3.21 & 19.27 & 1.36 & 42.03 & 2.09 & 64.92 & 2.51 & 70.40 & 3.58 & 111.03 & 1.79 & 48.26 & 355.9 & 1494.9 \\
\hline & 4.5 & 3.21 & 19.27 & 1.39 & 43.10 & 2.17 & 67.22 & 2.62 & 73.24 & 3.71 & 114.99 & 1.83 & 49.40 & 367.2 & 1542.3 \\
\hline & 3 & 3.21 & 19.27 & 1.43 & 44.14 & 2.20 & 68.34 & 2.67 & 74.08 & 3.78 & 117.18 & 1.88 & 50.76 & 374.5 & 1572.9 \\
\hline & 0 & 0.21 & 19.27 & 1.45 & 44.92 & 2.26 & 70.19 & 2.76 & 77.24 & 3.89 & 120.47 & 1.96 & 3.01 & 385.2 & 1617.9 \\
\hline \multicolumn{16}{|c|}{ Lupine season 2013/2014 } \\
\hline \multirow{4}{*}{$\begin{array}{l}1.2 \\
\text { Pan }\end{array}$} & 6 & 3.36 & 13.44 & 1.66 & 51.60 & 2.39 & 74.00 & 2.64 & 73.90 & 4.92 & 152.5 & 2.09 & 60.70 & 426.1 & 1789.8 \\
\hline & 4.5 & 3.36 & 13.44 & 1.74 & 53.90 & 2.49 & 77.10 & 2.81 & 78.80 & 5.08 & 157.6 & 2.17 & 62.80 & 443.6 & 1863.3 \\
\hline & 3 & 3.36 & 13.44 & 1.95 & 60.5 & 2.57 & 79.60 & 2.93 & 82.10 & 5.23 & 162.0 & 2.21 & 64.20 & 461.8 & 1939.7 \\
\hline & 0 & 3.36 & 13.44 & 2.00 & 62.0 & 2.61 & 1.0 & 3.05 &. .5 & 5.49 & 70.2 & 2.34 & 8.0 & 480.14 & 42016.6 \\
\hline \multicolumn{2}{|c|}{ Mean } & 3.36 & 13.44 & 1.84 & 57.0 & 2.52 & 77.90 & 2.86 & 80.08 & 5.18 & 160.58 & 2.20 & 63.93 & 452.96 & 61902.43 \\
\hline & 6 & 3.36 & 13.44 & 1.05 & 32.70 & 2.12 & 65.60 & 2.51 & 70.20 & 3.33 & 103.3 & 1.95 & 56.5 & 341.7 & 1435.3 \\
\hline 1.0 & 4.5 & 3.36 & 13.44 & 1.10 & 34.2 & 2.19 & 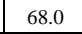 & 2.63 & 73.60 & 3.52 & 109.2 & 1.96 & 56.90 & 355.3 & 1492.4 \\
\hline Pan & 3 & 3.36 & 13.44 & 1.16 & 35.9 & 2.26 & 70.20 & 2.75 & 77.10 & 3.59 & 111.4 & 2.03 & 8.9 & 366.9 & 1541.2 \\
\hline & 0 & \begin{tabular}{|l}
3.36 \\
\end{tabular} & 13.44 & 1.19 & 37.00 & 2.35 & 73.00 & 2.94 & 82.30 & 3.74 & 115.8 & 2.12 & 1.50 & 383.0 & 1608.8 \\
\hline $\mathrm{Me}$ & ean & \begin{tabular}{|l}
3.36 \\
\end{tabular} & 13.44 & 1.13 & 34.95 & 2.23 & 69.20 & 2.71 & 75.80 & 3.55 & 109.9 & 2.02 & 58.5 & 361.77 & $7 \mid 1519.43$ \\
\hline & 6 & 3.36 & 13.44 & 1.03 & 32.00 & 1.76 & 54.50 & 2.26 & 63.40 & 2.75 & 85.40 & 1.11 & 32.20 & 280.9 & 1179.9 \\
\hline 0.8 & 4.5 & 3.36 & 13.44 & 1.06 & 32.80 & 1.82 & 56.50 & 2.43 & 68.10 & 2.84 & 88.00 & 1.16 & 33.70 & 292.5 & 1228.7 \\
\hline Pan. & 3 & \begin{tabular}{|l}
3.36 \\
\end{tabular} & 13.44 & 1.09 & 33.70 & 1.85 & 57.40 & 2.56 & 71.60 & 2.93 & 90.70 & 1.23 & 35.80 & 302.61 & $\begin{array}{lll}1271.1 \\
\end{array}$ \\
\hline & 0 & 3.36 & 13.44 & 1.13 & 35.00 & 1.94 & 60.20 & 2.68 & 74.90 & 3.03 & 94.00 & 1.27 & 36.90 & 314.4 & 1320.7 \\
\hline $\mathrm{Mer}$ & ean & 3.36 & 13.44 & 1.08 & 33.38 & 1.84 & 57.15 & 2.48 & 69.50 & 2.89 & 89.53 & 1.19 & 34.65 & 297.65 & $\begin{array}{ll}5 & 1250.1\end{array}$ \\
\hline 0 & 6 & 3.36 & 13.44 & 1.25 & 38.8 & 2.09 & +.1 & 2.47 & 69.17 & 3.67 & 113.7 & 1.72 & 49.80 & 349.6 & 1468.3 \\
\hline Mean for & 4.5 & 3.36 & 13.44 & 1.30 & 40.3 & 2.17 & 67.20 & 2.62 & 73.50 & 3.81 & 118.3 & 1.76 & 51.13 & 363.8 & 1528.13 \\
\hline
\end{tabular}

Egypt. J. Soil. Sci. 56, No. 2 (2016) 
\begin{tabular}{|l|c|c|c|c|c|c|c|c|c|c|c|c|c|c|c|}
\hline Bentonite & 3 & 3.36 & 13.44 & 1.40 & 43.4 & 2.23 & 69.07 & 2.75 & 76.93 & 3.92 & 121.4 & 1.82 & 52.97 & 377.1 & 1584.5 \\
\cline { 2 - 15 }
\end{tabular} \begin{tabular}{|l|l|l|l|l|l|l|l|l|l|l|l|l|l|l|}
\hline $\mathbf{0}$ & 3.36 & 13.44 & 1.44 & 44.7 & 2.30 & 71.40 & 2.88 & 80.9 & 4.09 & 126.7 & 1.91 & 55.50 & 392.5 & 1648.7 \\
\hline
\end{tabular} *sowing dates at first and at second season were 25/11/2012 and 30/11/2013.

**harvest dates at first and at second season were 28/4/2013 and 30/4/2014.

TABLE 5. Sesame daily, monthly and total actual evapotranspiration $\left(\mathbf{E T}_{\mathrm{a}, \mathrm{mm}}\right)$ as affected by different values of pan coefficient and bentonite conditioner rates.

\begin{tabular}{|c|c|c|c|c|c|c|c|c|c|c|c|}
\hline \multicolumn{12}{|c|}{ Sesame season 2013} \\
\hline \multicolumn{2}{|c|}{ months } & \multicolumn{2}{|c|}{ June ${ }^{*}$} & \multicolumn{2}{|c|}{ July } & \multicolumn{2}{|c|}{ August } & \multicolumn{2}{|c|}{ September $^{* *}$} & \multirow{2}{*}{\multicolumn{2}{|c|}{ Total }} \\
\hline Irrg. & \multirow{2}{*}{$\begin{array}{c}\text { Bent. } \\
\text { ton/fed. }\end{array}$} & \multirow{2}{*}{$\frac{\text { Daily }}{\mathrm{mm}}$} & \multirow{2}{*}{$\begin{array}{c}\text { monthly } \\
\mathrm{mm} \\
\end{array}$} & \multirow{2}{*}{\begin{tabular}{|c} 
Daily \\
$\mathrm{mm}$
\end{tabular}} & \multirow{2}{*}{$\frac{\text { monthly }}{\mathrm{mm}}$} & \multirow{2}{*}{$\begin{array}{c}\text { Daily } \\
\mathrm{mm} \\
\end{array}$} & \multirow{2}{*}{$\begin{array}{c}\text { monthly } \\
\mathrm{mm}\end{array}$} & \multirow{2}{*}{$\begin{array}{c}\text { Daily } \\
\mathrm{mm} \\
\end{array}$} & \multirow{2}{*}{$\begin{array}{c}\text { monthly } \\
\mathrm{mm}\end{array}$} & & \\
\hline Regimef & & & & & & & & & & mm & $m^{3}$ fed $^{-1}$ \\
\hline \multirow{4}{*}{$\begin{array}{l}1.2 \\
\text { Pan }\end{array}$} & 6 & 3.77 & 97.96 & 8.35 & 258.7 & 8.36 & 259.2 & 4.92 & 133 & 748.9 & 3145.2 \\
\hline & 4.5 & 3.78 & 98.38 & 8.60 & 266.7 & 8.71 & 269.9 & 5.11 & 138 & 773.1 & 3247.0 \\
\hline & 3 & 3.79 & 98.51 & 8.91 & 276.2 & 8.99 & 278.7 & 5.32 & 143.6 & 797.1 & 3347.6 \\
\hline & 0 & 3.80 & 98.77 & 9.10 & 282 & 9.38 & 290.7 & 5.50 & 148.4 & 819.9 & 3443.7 \\
\hline Mean & & 3.79 & 98.41 & 8.74 & 270.90 & 8.86 & 274.63 & 5.21 & 140.75 & 784.75 & 3295.9 \\
\hline \multirow{4}{*}{$\begin{array}{c}1.0 \\
\text { Pan. }\end{array}$} & 6 & 3.75 & 98.86 & 7.2 & 249.2 & 7.39 & 254.5 & 5.04 & 158 & 760.5 & 3194.2 \\
\hline & 4.5 & 3.77 & 98.12 & 7.48 & 241.1 & 7.75 & 244.7 & 5.24 & 147.7 & 731.5 & 3072.4 \\
\hline & 3 & 3.77 & 97.98 & 7.78 & 231.8 & 7.89 & 240.2 & 5.47 & 141.6 & 711.6 & 2988.7 \\
\hline & 0 & 3.80 & 97.41 & 8.04 & 223.1 & 8.21 & 229.2 & 5.85 & 136.1 & 685.9 & 2880.6 \\
\hline Mean & & 3.77 & 98.09 & 7.63 & 236.30 & 7.81 & 242.15 & 5.40 & 145.85 & 722.38 & 3034.0 \\
\hline \multirow{4}{*}{$\begin{array}{c}0.8 \\
\text { Pan }\end{array}$} & 6 & 3.74 & 97.31 & 5.58 & 173.1 & 6.61 & 204.9 & 4.96 & 133.8 & 609.1 & 2558.0 \\
\hline & 4.5 & 3.75 & 97.46 & 5.73 & 177.6 & 6.83 & 211.6 & 5.17 & 139.6 & 626.3 & 2630.5 \\
\hline & 3 & 3.76 & 97.75 & 5.96 & 184.6 & 7.07 & 219.3 & 5.27 & 142.4 & 644 & 2704.9 \\
\hline & 0 & 3.77 & 98.13 & 6.12 & 189.6 & 7.17 & 222.3 & 5.36 & 144.8 & 654.8 & 2750.2 \\
\hline Mean & & 3.76 & 97.66 & 5.85 & 181.23 & 6.92 & 214.53 & 5.19 & 140.15 & 633.55 & 2660.9 \\
\hline \multirow{4}{*}{$\begin{array}{l}\text { Overall mean } \\
\text { For Bentonite }\end{array}$} & 6 & 3.75 & 97.56 & 7.04 & 218.30 & 7.45 & 231.10 & 4.97 & 134.30 & 681.30 & 2861.3 \\
\hline & 4.5 & 3.76 & 97.94 & 7.27 & 225.37 & 7.76 & 240.57 & 5.17 & 139.73 & 703.67 & 2955.4 \\
\hline & 3 & 3.77 & 98.13 & 7.75 & 240.27 & 7.99 & 247.57 & 5.35 & 141.45 & 757.37 & 3181.0 \\
\hline & 0 & 3.79 & 98.59 & 8.62 & 267.30 & 8.25 & 255.83 & 5.57 & 150.40 & 745.07 & 3129.4 \\
\hline \multicolumn{12}{|c|}{ Sesame season 2014} \\
\hline 1.2 & 6 & 3.60 & 108.0 & 8.70 & 269.6 & 8.74 & 270.8 & 5.45 & 125.3 & 773.7 & 3249.5 \\
\hline \multirow[t]{3}{*}{ Pan } & 4.5 & 3.76 & 112.8 & 9.03 & 280 & 9.13 & 283.1 & 5.66 & 130.1 & 806.0 & 3385.2 \\
\hline & 3 & 3.78 & 113.4 & 9.39 & 291.1 & 9.55 & 295.4 & 5.93 & 136.4 & 836.3 & 3512.5 \\
\hline & 0 & 3.82 & 114.6 & 9.58 & 297.1 & 9.68 & 300.1 & 6.17 & 141.8 & 853.6 & 3585.1 \\
\hline Mean & & 3.74 & 112.2 & 9.175 & 284.45 & 9.27 & 287.35 & 5.8025 & 133.4 & 817.4 & 3433.1 \\
\hline 1.0 & 6 & 3.47 & 104.2 & 7.49 & 232.2 & 7.58 & 234.9 & 5.53 & 127.3 & 698.6 & 2934.1 \\
\hline \multirow[t]{3}{*}{ Pan } & 4.5 & 3.59 & 107.8 & 7.79 & 241.4 & 8.08 & 250.6 & 5.80 & 133.5 & 733.3 & 3079.9 \\
\hline & 3 & 3.66 & 109.9 & 8.16 & 253.1 & 8.35 & 258.8 & 6.10 & 140.3 & 762.1 & 3200.8 \\
\hline & 0 & 3.72 & 111.7 & 8.45 & 262 & 8.61 & 266.9 & 6.55 & 150.7 & 791.3 & 3323.5 \\
\hline Mean & & 3.61 & 108.4 & 7.9725 & 247.175 & 8.155 & 252.8 & 5.995 & 137.95 & 746.33 & 3134.6 \\
\hline 0.8 & 6 & 3.40 & 102 & 5.83 & 180.7 & 6.78 & 210.2 & 5.48 & 126.1 & 619 & 2599.8 \\
\hline Pan & 4.5 & 3.51 & 105.2 & 5.95 & 184.5 & 7.01 & 217.3 & 5.72 & 131.6 & 638.6 & 2682.1 \\
\hline & 3 & 3.58 & 107.5 & 6.19 & 191.9 & 7.21 & 223.5 & 5.83 & 134.2 & 657.1 & 2759.8 \\
\hline & 0 & 3.60 & 108 & 6.70 & 207.6 & 7.37 & 228.6 & 5.98 & 137.6 & 681.8 & 2863.6 \\
\hline Mean & & 3.52 & 105.68 & 6.17 & 191.18 & 7.09 & 219.90 & 5.75 & 132.38 & 649.13 & 2726.3 \\
\hline Overall mean & 6 & 3.49 & 104.73 & 7.34 & 227.50 & 7.70 & 238.63 & 5.49 & 126.23 & 697.10 & 2927.8 \\
\hline For Bentonite & 4.5 & 3.54 & 108.60 & 7.59 & 235.30 & 8.08 & 250.33 & 5.73 & 131.73 & 725.97 & 3049.1 \\
\hline
\end{tabular}

Egypt. J. Soil. Sci. 56, No. 2 (2016) 


\begin{tabular}{|l|c|c|c|c|c|c|c|c|c|c|c|}
\hline & 3 & 3.67 & 110.27 & 7.91 & 245.37 & 8.36 & 259.23 & 5.96 & 136.97 & 751.81 & 3157.6 \\
\cline { 2 - 10 } & 0 & 3.71 & 111.43 & 8.24 & 255.57 & 8.55 & 265.20 & 6.23 & 143.37 & 775.57 & 3257.40 \\
\hline
\end{tabular}

**harvest dates at first and at second season were 28/9/2013 and 24/9/2014

In addition, the corresponding values for the second season were 817.4, 746.33 and $649.14 \mathrm{~mm}$ for the same respective irrigation regime. In general, the highest actual evapotranspiration was recorded under 1.2 accumulative pan evaporation treatment, while the lowest one recorded under 0.8 irrigation regimes. From these results, it could be concluded that the quantity of water use depends on the frequent irrigation intervals. When soil kept wet by short irrigation intervals, higher seasonal consumptive use was obtained (Uacn et al., 2007).

Regarding to the effect of bentonite rates, data presented in Table (5) showed that average values of actual evapotranspiration in first season were 745.1, $757.37,703.67$ and $681.30\left(6.0,4.5,3.0\right.$ and zero ton fed $\left.^{-1}\right)$ under bentonite application rates, respectively. While in the second season, results indicated that values were 775.57, 751.8, 725.97 and 697.09 for the same respective bentonite treatments. The highest and lowest values were recorded when applied 6.0 and zero ton $\mathrm{fed}^{-1}$ bentonite, respectively.

Comparison between calculated various potential evapotranspiration methods $\left(E T_{p}\right)$ for both crops

Lupin crop

The methods used to estimate reference potential evapotranspiration (for cool season clipped grass) depend on the availability of accurate meteorological data and on local practices. In FAO modified Penman method (Doorenbos and Pruitt, 1977), the reference crop was defined as: An extended surface of 0.08 to $0.15 \mathrm{~mm}$ tall green grass cover of uniform height, actively growing, completely shading the ground, and not short of water and the Penman-Monteith method. The PM "default" method (Allen et al., 1998) equation has been standardized to estimate the reference ET of $12-\mathrm{cm}$ tall, cool-season grass and $50 \mathrm{~cm}$ tall alfalfa need a full range of accurate meteorological data. If, however, data on humidity, wind speed and radiation are missing the Hargreaves-Samani (1985) method is recommended.

The various methods of determining potential evapotranspiration are based on one or more of these requirements. Calculations of lupine crop evapotranspiration values $\left(\mathrm{ET}_{\mathrm{c}}\right)$ involved the use of potential evapotranspiration values $\left(\mathrm{ET}_{\mathrm{o}}\right)$ and crop coefficient values $\left(\mathrm{k}_{\mathrm{c}}\right)$ are presented in Table 6 . The $\left(\mathrm{ET}_{\mathrm{o}}\right)$ values were calculated by five methods; namely pan evaporation method presented by Doorenbos and Pruitt (1977) still the most practical and accurate meteorological method for determining irrigation water requirements (Stanhill, 2002), modified Penman method presented by Doorenbos and Kassam (1979), 1985 Hargreaves reference evapotranspiration equation, modified PenmanMontieth method presented by Allen et al. (1998) and FAO modified of BlaneyCriddle method has received considerable interest; it is a temperature-based method, according to Cuenca and Amegee (1987). 
The duration of lupine crop growth stages are 15, 60, 55 and 24 days for the initial, crop development, mid season and late-season, respectively. Average crop coefficient values $\left(\mathrm{K}_{\mathrm{c}}\right)$ are calculated by Doorenbos and Kassam (1979). These values are $0.5,0.8,1.15$ and 0.7 for the previous stages. The data show that, the highest obtained $\left(\mathrm{ET}_{\mathrm{c}}, \mathrm{mm}\right)$ values were during March. The differences between the values of five formulae are non- big, so, we prefer to use pan evaporation and 1985 Hargreaves reference evapotranspiration equation than the other three methods for calculation of lupine $\mathrm{ET}_{\mathrm{c}}$ in Ismailia area. In this connection, it could be mentioned here that pan evaporation and 1985 Hargreaves reference evapotranspiration equations are the best under Ismailia conditions and it can be used for scheduling irrigation for lupine crop. These results are in the line with respected by Doorenbos and Kassam (1979).

TABLE 6. Lupine daily, monthly and total evapotranspiration $\left(\mathbf{E T}_{\mathrm{c}}, \mathbf{m m}\right)$ calculated by different methods during the two years.

\begin{tabular}{|c|c|c|c|c|c|c|c|c|c|c|c|c|c|c|}
\hline \multicolumn{15}{|c|}{ Winter season 2012/2013 } \\
\hline \multirow{3}{*}{ equation } & \multicolumn{2}{|c|}{ Nov." } & \multicolumn{2}{|c|}{ Dec. } & \multicolumn{2}{|c|}{ Jan. } & \multicolumn{2}{|c|}{ Feb. } & \multicolumn{2}{|c|}{ Mar. } & \multicolumn{2}{|c|}{ Apr. ${ }^{* *}$} & \multirow{2}{*}{\multicolumn{2}{|c|}{ Total }} \\
\hline & \multirow{2}{*}{\begin{tabular}{|c|} 
Daily \\
$\mathrm{mm}$ \\
\end{tabular}} & \multirow{2}{*}{\begin{tabular}{|c|} 
monthly \\
$\mathrm{mm}$ \\
\end{tabular}} & \multirow{2}{*}{\begin{tabular}{|l|} 
Daily \\
$\mathrm{mm}$ \\
\end{tabular}} & \multirow{2}{*}{\begin{tabular}{|c|} 
monthly \\
$\mathrm{mm}$ \\
\end{tabular}} & \multirow{2}{*}{\begin{tabular}{|l|} 
Daily \\
$\mathrm{mm}$ \\
\end{tabular}} & \multirow{2}{*}{\begin{tabular}{|c|} 
monthly \\
$\mathrm{mm}$ \\
\end{tabular}} & \multirow{2}{*}{\begin{tabular}{|l} 
Daily \\
mm \\
\end{tabular}} & \multirow{2}{*}{\begin{tabular}{|c} 
monthly \\
$\mathrm{mm}$ \\
\end{tabular}} & \multirow{2}{*}{\begin{tabular}{|l} 
Daily \\
$\mathrm{mm}$ \\
\end{tabular}} & \multirow{2}{*}{\begin{tabular}{|c|} 
monthly \\
$\mathrm{mm}$ \\
\end{tabular}} & \multirow{2}{*}{\begin{tabular}{|l} 
Daily \\
$\mathrm{mm}$ \\
\end{tabular}} & \multirow{2}{*}{\begin{tabular}{|c|} 
monthly \\
$\mathrm{mm}$ \\
\end{tabular}} & & \\
\hline & & & & & & & & & & & & & $\mathbf{m m}$ & $\mathrm{m}^{3}$ Fed $^{-1}$ \\
\hline $\mathbf{E}_{\text {pan }}$ & 1.17 & 5.85 & 1.28 & 39.75 & 1.45 & 44.95 & 2.57 & 71.94 & 3.99 & 123.7 & 3.24 & 90.86 & 377.0 & 1583 \\
\hline P-M & 1.30 & 6.48 & 1.84 & 57.15 & 2.04 & 63.17 & 3.42 & 95.62 & 4.95 & 153.3 & 4.14 & 115.95 & 491.7 & 2065 \\
\hline H.equ. & 1.25 & 6.25 & 1.40 & 43.54 & 1.70 & 52.68 & 2.88 & 80.51 & 4.28 & 132.62 & 4.06 & 113.7 & 429.3 & 1803 \\
\hline Mod.Pen. & 1.85 & 9.25 & 2.18 & 67.65 & 2.32 & 71.92 & 3.75 & 105.09 & 5.10 & 157.95 & 4.74 & 132.67 & 544.5 & 2287 \\
\hline B\&C & 2.51 & 12.55 & 2.29 & 71.00 & 2.48 & 76.88 & 3.81 & 106.56 & 5.23 & 162.2 & 4.82 & 134.86 & 567.07 & 2382 \\
\hline \multicolumn{15}{|c|}{ Winter season 2013/2014 } \\
\hline $\mathbf{E}_{\mathrm{pan}}$ & 1.17 & 3.51 & 1.24 & 38.59 & 1.45 & 44.95 & 2.51 & 70.36 & 3.99 & 123.69 & 3.35 & 100.36 & 381.46 & 1602 \\
\hline P-M & 1.3 & 3.89 & 1.79 & 55.49 & 2.04 & 63.17 & 3.34 & 93.52 & 4.95 & 153.3 & 4.27 & 128.07 & 497.44 & 2089 \\
\hline H.equ. & 1.25 & 3.75 & 1.36 & 42.27 & 1.70 & 52.68 & 2.81 & 78.74 & 4.28 & 132.62 & 4.19 & 125.63 & 435.7 & 1830 \\
\hline Mod.Pen. & 1.85 & 5.55 & 2.12 & 65.68 & 2.32 & 71.92 & 3.67 & 102.8 & 5.10 & 157.9 & 4.88 & 146.54 & 550.4 & 2312 \\
\hline$B \& C$ & 2.51 & 7.53 & 2.22 & 68.94 & 2.48 & 76.88 & 3.72 & 104.22 & 5.23 & 162.22 & 4.97 & 148.96 & 568.8 & 2389 \\
\hline
\end{tabular}

* sowing dates at first and at second season were 5/6/2013 and 1/6/2014.

**harvest dates at first and at second season were 28/9/2013 and 24/9/2014.

\section{Sesame crop}

Sesame crop potential evapotranspiration values, $\mathrm{ET}_{\mathrm{p}}$ were determined for each formula during the two growing seasons. The obtained data are given in Table 7. These values were high compared with the sesame crop potential evapotranspiration values, determined by different formula. Five methods were involved in calculated $\mathrm{ET}_{\mathrm{o}}$. They were pan evaporation method, modified Penman, Hargreaves, Penman-Montieth and Blaney-Criddle method. Crop coefficient values given by Doorenbos and Kassam (1979) are 0.35, 0.75, 1.1 and 0.25 for the forth or four sesame stages. These stages are: initial stage (20 days) developed stage (30 days) mid-season (43 day) and late-season ( 25 days). The obtained results presented in Table 7 show that sesame crop reference evapotranspiration varied from 463.54 to 699.95 and from 468.1 to $709.02 \mathrm{~mm}$ Egypt. J. Soil. Sci. 56, No. 2 (2016) 
for first and second season, respectively, depending on formulae used to calculate the potential crop evapotranspiration. It is noticed that $\mathrm{ET}_{\mathrm{a}}$ values are greater than the calculated $\mathrm{ET}_{\mathrm{c}}$ for 1.2 and 1.0 accumulative evaporation pan, but lower than 0.8 accumulative evaporation pan. However, the nearest $\mathrm{ET}_{\mathrm{a}}$ values to the $\mathrm{ET}_{\mathrm{c}}$ values were obtained from the Blaney-Cridde method.

TABLE 7. Sesame daily, monthly and total evapotranspiration $\left(\mathbf{E T}_{\mathbf{c}}, \mathbf{m m}\right)$ calculated by different methods during the two years of studying sesame crop.

\begin{tabular}{|c|c|c|c|c|c|c|c|c|c|c|}
\hline \multicolumn{11}{|c|}{ Sesame season 2013} \\
\hline \multirow{3}{*}{ equation } & \multicolumn{2}{|c|}{ June $^{*}$} & \multicolumn{2}{|c|}{ July } & \multicolumn{2}{|c|}{ August } & \multicolumn{2}{|c|}{ September ${ }^{* *}$} & \multirow{2}{*}{\multicolumn{2}{|c|}{ Total }} \\
\hline & \multirow{2}{*}{$\begin{array}{c}\text { Daily } \\
\text { mm }\end{array}$} & \multirow{2}{*}{$\begin{array}{c}\text { monthly } \\
\text { mm }\end{array}$} & \multirow{2}{*}{$\begin{array}{c}\text { Daily } \\
\mathbf{m m}\end{array}$} & \multirow{2}{*}{\begin{tabular}{|c|} 
monthly \\
mm \\
\end{tabular}} & \multirow{2}{*}{$\begin{array}{c}\text { Daily } \\
\text { mm }\end{array}$} & \multirow{2}{*}{$\begin{array}{c}\text { monthly } \\
\text { mm }\end{array}$} & \multirow{2}{*}{$\begin{array}{c}\text { Daily } \\
\text { mm }\end{array}$} & \multirow{2}{*}{$\begin{array}{c}\text { monthly } \\
\text { mm }\end{array}$} & & \\
\hline & & & & & & & & & $\mathbf{m m}$ & $\mathrm{m}^{3} \mathrm{Fed}^{-1}$ \\
\hline $\mathbf{E}_{\mathrm{pan}}$ & 2.46 & 61.45 & 4.92 & 152.61 & 6.18 & 191.64 & 2.07 & 57.84 & 463.54 & 1947 \\
\hline P-M & 2.83 & 70.74 & 5.48 & 169.85 & 6.97 & 216.19 & 2.39 & 66.79 & 523.57 & 2199 \\
\hline H.equ. & 2.75 & 68.71 & 5.41 & 167.82 & 6.50 & 201.53 & 2.06 & 57.73 & 495.79 & 2082 \\
\hline Mod.Pen. & 3.18 & 79.55 & 7.11 & 220.55 & 8.93 & 276.89 & 2.71 & 75.75 & 652.66 & 2741 \\
\hline B\&C & 3.92 & 97.95 & 7.33 & 227.36 & 9.37 & 290.53 & 3.00 & 84.11 & 699.95 & 2940 \\
\hline \multicolumn{11}{|c|}{ Sesame season 2014} \\
\hline $\mathbf{E}_{\text {pan }}$ & 2.62 & 78.61 & 5.19 & 161.04 & 6.18 & 191.64 & 1.53 & 36.81 & 468.1 & 1966 \\
\hline P-M & 3.02 & 90.48 & 5.78 & 179.23 & 6.97 & 216.19 & 1.77 & 42.50 & 528.4 & 2219 \\
\hline H.equ. & 2.93 & 87.88 & 5.71 & 177.09 & 6.50 & 201.53 & 1.53 & 36.74 & 503.24 & 2114 \\
\hline Mod.Pen. & 3.39 & 101.75 & 7.51 & 232.37 & 8.93 & 276.89 & 2.01 & 48.20 & 659.57 & 2770 \\
\hline B\&C & 4.18 & 125.28 & 7.73 & 239.68 & 9.37 & 290.53 & 2.23 & 53.53 & 709.02 & 2978 \\
\hline
\end{tabular}

*sowing dates at first and at second season were 5/6/2013 and 1/6/2014

**harvest dates at first and at second season were 28/9/2013 and 24/9/2014.

Irrigation schedule under different soil conditioner rates

Lupine yield

Generally, growth parameters are influenced by the irrigation scheduling using pan coefficient and rates of bentonite soil conditioner. The obtained data are presented in Table 8. Results showed that the effects of irrigation on two studied characters (i.e. straw and grains yield) were significant in both seasons. The highest values of two studied characters were gained from the 1.2 pan coefficient irrigation treatment followed by the other irrigation treatments, and the differences were significant. The lowest values for two studied characters were produced from the irrigation treatment at 0.8 pan coefficient. The highest values of straw yield (ton fed $\left.{ }^{-1}\right)$ and grain yield $\left(\mathrm{kg} \mathrm{fed}^{-1}\right)$ were $2.29,6.58$ and 1678 in the first season and 2.48, 6.64 and 999 in the second season, respectively. These results agreed with El-Fahal (2007).

Regarding the effect of bentonite soil conditioner, results indicated that the effect of bentonite soil conditioner were significant on two studied growth characters (straw and grains) in both seasons. Also, bentonite rate of 6.0 ton $\mathrm{kg}$ $\mathrm{fed}^{-1}$ gave the highest values of straw and grain yield, and the values were 1.8 , 5.59 and 838 in the first season and 1.91, 5.76 and 864 in the second season, respectively. Similar results were reported by Croker et al. (2004) and Noble et al. (2001) who reported that soil amendment with high CEC materials such as high activity clay minerals (e.g. bentonite) increased CEC and improved plant biomass in sand soils. Moreover, growth parameters of crops as well as their seed yields were beneficially increased with increasing the rate of applied conditioner (Hassan and Mahmoud, 2013). 
The interaction between irrigation treatments and bentonite conditioner rates were significant for two studied growth season. Data reveal that the highest yield for lupine yield (straw and grains) was recorded under 1.2 pan irrigation treatment and applied of 6.0 ton $\mathrm{fed}^{-1}$ bentonite for both growth seasons as compared to either treatments.

TABLE 8. Effect of irrigation scheduling using different pan and different rates of bentonite soil conditioner on lupine yield.

\begin{tabular}{|c|c|c|c|c|c|c|c|}
\hline \multirow[b]{2}{*}{$\begin{array}{l}\text { Irrg. } \\
\text { Regime }\end{array}$} & \multirow[b]{2}{*}{$\begin{array}{l}\text { Bent. Rates } \\
\text { ton /fed. }\end{array}$} & \multicolumn{3}{|c|}{ Season 2012-2013 } & \multicolumn{3}{|c|}{ Season 2013-2014 } \\
\hline & & $\begin{array}{c}\text { Straw } \\
\left(\text { ton } \text { fed }^{-1}\right)\end{array}$ & $\begin{array}{c}\text { grain } \\
\text { ardb* fed }_{1}^{-} \\
\text {f f }^{-}\end{array}$ & $\begin{array}{c}\text { grain } \\
\text { Kg fed }^{-1}\end{array}$ & $\mid \begin{array}{c}\text { Straw } \\
\left(\text { ton }_{\text {fed }}{ }^{-1}\right)\end{array}$ & $\begin{array}{c}\text { grain } \\
\operatorname{ardb}^{*} \text { fed }^{-1}\end{array}$ & $\begin{array}{c}\text { grain } \\
\text { Kg fed }^{-1}\end{array}$ \\
\hline \multirow{4}{*}{1.2 pan. } & 6.0 & $2.89 a$ & $7.16 \mathrm{a}$ & 1075 & $3.11 \mathrm{a}$ & $7.40 \mathrm{a}$ & $1111 \mathrm{a}$ \\
\hline & 4.5 & $2.28 \mathrm{~b}$ & $6.73 b$ & 1010 & $2.95 b$ & $6.66 \mathrm{~b}$ & $999 b$ \\
\hline & 3.0 & $2.10 \mathrm{c}$ & $6.38 \mathrm{c}$ & 958 & $1.95 \mathrm{c}$ & $6.36 c$ & $954 b c$ \\
\hline & 0.0 & $1.89 \mathrm{~d}$ & $6.04 d$ & 907 & $1.91 \mathrm{c}$ & $6.12 \mathrm{c}$ & $919 d$ \\
\hline \multicolumn{2}{|c|}{ Mean for irrigation } & $2.29 \mathrm{a}$ & $6.58 \mathrm{a}$ & 1678 & $2.48 \mathrm{a}$ & $6.64 a$ & $999 a$ \\
\hline \multirow{4}{*}{1.0 pan. } & 6.0 & $1.64 \mathrm{e}$ & $6.07 \mathrm{~d}$ & 910 & $1.72 \mathrm{~d}$ & $6.36 \mathrm{c}$ & $955 \mathrm{~cd}$ \\
\hline & 4.5 & $1.49 \mathrm{f}$ & $5.58 \mathrm{e}$ & 837 & $1.43 \mathrm{e}$ & $5.62 d$ & $844 \mathrm{e}$ \\
\hline & 3.0 & $1.19 \mathrm{~g}$ & $5.14 \mathrm{f}$ & 771 & $1.30 \mathrm{f}$ & $5.11 \mathrm{e}$ & $767 \mathrm{f}$ \\
\hline & 0.0 & $1.11 \mathrm{~g}$ & $4.45 \mathrm{~g}$ & 668 & $1.29 \mathrm{f}$ & $4.52 \mathrm{f}$ & $678 \mathrm{~g}$ \\
\hline \multicolumn{2}{|c|}{ Mean for irrigation } & $1.36 \mathrm{~b}$ & $5.31 \mathrm{~b}$ & 797 & $1.44 \mathrm{~b}$ & $5.40 \mathrm{~b}$ & $811 \mathrm{~b}$ \\
\hline \multirow{4}{*}{0.8 pan. } & 6.0 & $0.87 \mathrm{~h}$ & $3.53 \mathrm{~h}$ & 530 & $0.88 \mathrm{~g}$ & $3.51 \mathrm{~g}$ & $527 \mathrm{~h}$ \\
\hline & 4.5 & $0.73 \mathrm{i}$ & $3.13 \mathrm{i}$ & 470 & $0.77 \mathrm{gh}$ & $3.24 \mathrm{~h}$ & $486 \mathrm{i}$ \\
\hline & 3.0 & $0.70 \mathrm{i}$ & $3.04 \mathrm{i}$ & 456 & $0.71 \mathrm{~h}$ & $2.98 \mathrm{i}$ & $447 j$ \\
\hline & 0.0 & $0.67 \mathrm{i}$ & $2.98 \mathrm{i}$ & 448 & $0.66 \mathrm{~h}$ & $2.83 \mathrm{i}$ & $425 j$ \\
\hline \multicolumn{2}{|c|}{ Mean for irrigation } & $0.76 \mathrm{c}$ & $3.17 \mathrm{c}$ & 476 & $0.75 \mathrm{c}$ & $3.14 \mathrm{c}$ & $471 \mathrm{c}$ \\
\hline \multicolumn{8}{|c|}{ Mean for bent. Conditioner } \\
\hline \multicolumn{2}{|c|}{6.0 ton fed..$^{-1}$} & $1.80 \mathrm{a}$ & $5.59 \mathrm{a}$ & 838 & $1.91 \mathrm{a}$ & $5.76 a$ & $864 a$ \\
\hline \multicolumn{2}{|c|}{4.5 tonfed. $^{-1}$} & $1.50 \mathrm{~b}$ & $5.15 b$ & 1793 & $1.72 b$ & $5.17 \mathrm{~b}$ & $776 b$ \\
\hline \multicolumn{2}{|c|}{3.0 ton fed. $^{-1}$} & $1.33 \mathrm{c}$ & $4.85 \mathrm{c}$ & 628 & $1.32 \mathrm{c}$ & $4.82 \mathrm{c}$ & $726 c$ \\
\hline \multicolumn{2}{|c|}{0.0 ton fed. $^{-1}$} & $1.24 \mathrm{~d}$ & $4.49 \mathrm{~d}$ & 674 & $1.28 \mathrm{c}$ & $4.49 \mathrm{~d}$ & $674 d$ \\
\hline
\end{tabular}

$* \mathrm{Ardb}=150 \mathrm{~kg}$

\section{Sesame yield}

Sesame yield (seeds and straw) as influenced by various treatments, i.e. irrigation scheduling and bentonite rates are presented in Table 9. Statistical analysis of the variance demonstrated that irrigation and bentonite rates had a significant effect upon the productivity of sesame in both seasons. The highfrequency schedule (1.2 pan coefficient) yielded the maximum seed and straw yield followed by the other irrigation treatments. The differences in straw yield of sesame between the irrigation treatments (i.e. 1.2 and 1.0 pan) were found to be insignificant as well as the previous irrigation pan treatments and the 0.8 pan were significant. Whereas, the differences in seed yield of sesame between the irrigation treatments, i.e. $1.2,1.0$ and 0.8 pan coefficient were found to be significant. These results are in harmony with those reported by Sarhadi and Sharif (2014). 
Regarding the role of bentonite as a soil conditioner on yield of sesame, results in Table 9 revealed that increasing bentonite rate resulted in a significant increase in sesame yield. Such results prove that maintaining soil moisture at high level by both increasing frequency of irrigation water and bentonite rates maximized seed sesame production. Decreasing soil moisture beyond such level decreased yield production of sesame in sandy soils. This trend may be due to the multiple effects of water stress on plant growth, which in turn reflected on seed yield. These results are in agreement with those reported by Jouyban and Moosavi (2011).

TABLE 9. Effect of irrigation scheduling using pan and rates of bentonite soil conditioner on sesame yield.

\begin{tabular}{|c|c|c|c|c|c|c|c|}
\hline \multirow{2}{*}{$\begin{array}{l}\text { Irrg. } \\
\text { Regime }\end{array}$} & \multirow{2}{*}{$\begin{array}{c}\text { Bent. Rates } \\
\text { ton /fed. }\end{array}$} & \multicolumn{3}{|c|}{ Season 2013} & \multicolumn{3}{|c|}{ Season 2014} \\
\hline & & $\begin{array}{c}\text { Straw } \\
{\text { ton } \text { fed }^{-1}}^{-1}\end{array}$ & $\begin{array}{c}\text { Seed } \\
\text { Ardb* }^{*} \\
\text { fed }^{-1}\end{array}$ & $\begin{array}{c}\text { Seed } \\
\text { Kg fed }^{-1}\end{array}$ & $\begin{array}{c}\text { Straw } \\
{\text { ton } \text { fed }^{-1}}^{-1}\end{array}$ & $\begin{array}{c}\text { Seed } \\
\text { Ardb* }^{*} \\
\text { fed }^{-1}\end{array}$ & $\begin{array}{c}\text { Seed } \\
\text { Kg fed }^{-1}\end{array}$ \\
\hline \multirow{4}{*}{1.2 pan. } & 6.0 & $1.29 \mathrm{a}$ & $6.13 \mathrm{a}$ & $736 a$ & $1.16 \mathrm{a}$ & $5.83 a$ & $700 \mathrm{a}$ \\
\hline & 4.5 & $0.93 \mathrm{c}$ & $5.40 \mathrm{~b}$ & $648 b$ & $1.01 \mathrm{bc}$ & $5.10 \mathrm{bc}$ & $612 b c$ \\
\hline & 3.0 & $0.87 \mathrm{~d}$ & $4.97 \mathrm{c}$ & $596 \mathrm{c}$ & $0.87 \mathrm{de}$ & $4.87 \mathrm{c}$ & $584 \mathrm{~cd}$ \\
\hline & 0.0 & $0.79 \mathrm{ef}$ & $4.50 \mathrm{e}$ & $540 \mathrm{e}$ & $0.80 \mathrm{ef}$ & $4.50 \mathrm{~d}$ & $540 \mathrm{de}$ \\
\hline \multicolumn{2}{|c|}{ Mean for irrigation } & $0.97 \mathrm{a}$ & $5.25 \mathrm{a}$ & $630 \mathrm{a}$ & $0.96 \mathrm{a}$ & $5.08 \mathrm{a}$ & $609 a$ \\
\hline \multirow{4}{*}{1.0 pan. } & 6.0 & $1.10 \mathrm{~b}$ & $5.57 \mathrm{~b}$ & $668 b$ & $1.08 \mathrm{~b}$ & $5.27 \mathrm{~b}$ & $632 b$ \\
\hline & 4.5 & $0.93 \mathrm{c}$ & $4.70 \mathrm{~d}$ & $564 d$ & $0.94 \mathrm{~cd}$ & $4.50 \mathrm{~d}$ & $540 \mathrm{~b}-\mathrm{e}$ \\
\hline & 3.0 & $0.83 \mathrm{de}$ & $4.10 \mathrm{f}$ & $492 \mathrm{f}$ & $0.84 \mathrm{e}$ & $4.27 \mathrm{de}$ & 512ef \\
\hline & 0.0 & $0.80 \mathrm{ef}$ & $3.67 \mathrm{~g}$ & $440 \mathrm{~g}$ & $0.80 \mathrm{ef}$ & $3.97 \mathrm{e}$ & $476 f$ \\
\hline \multicolumn{2}{|c|}{ Mean for irrigation } & $0.92 \mathrm{~b}$ & $4.51 \mathrm{~b}$ & $541 b$ & $0.92 b$ & $4.50 \mathrm{~b}$ & $540 \mathrm{~b}$ \\
\hline \multirow{4}{*}{0.8 pan. } & 6.0 & $0.80 \mathrm{ef}$ & $2.50 \mathrm{~h}$ & $300 \mathrm{~h}$ & $0.84 \mathrm{e}$ & $2.60 \mathrm{f}$ & $312 \mathrm{~g}$ \\
\hline & 4.5 & $0.76 \mathrm{f}$ & $2.30 \mathrm{i}$ & $276 \mathrm{i}$ & $0.73 \mathrm{fg}$ & $2.30 \mathrm{fg}$ & $276 \mathrm{~h}$ \\
\hline & 3.0 & $0.67 \mathrm{~g}$ & $2.20 \mathrm{i}$ & $264 \mathrm{i}$ & $0.66 \mathrm{gh}$ & $2.10 \mathrm{~g}$ & $252 \mathrm{~h}$ \\
\hline & 0.0 & $0.55 \mathrm{~h}$ & $1.87 \mathrm{j}$ & $224 \mathrm{~g}$ & $0.59 \mathrm{~h}$ & $2.07 \mathrm{~g}$ & $248 \mathrm{~h}$ \\
\hline \multicolumn{2}{|c|}{ Mean for irrigation } & $0.70 \mathrm{c}$ & $2.22 \mathrm{c}$ & $266 \mathrm{c}$ & $0.71 \mathrm{c}$ & $2.70 \mathrm{c}$ & $280 \mathrm{c}$ \\
\hline \multicolumn{8}{|c|}{ Mean for bent. conditioner } \\
\hline \multicolumn{2}{|c|}{6.0 ton fed. $^{-1}$} & $1.07 \mathrm{a}$ & $4.73 a$ & $568 \mathrm{a}$ & $1.03 \mathrm{a}$ & $4.66 \mathrm{a}$ & $559 a$ \\
\hline \multicolumn{2}{|l|}{4.5 tonfed. $^{-1}$} & $0.87 \mathrm{~b}$ & $4.13 b$ & $496 b$ & $0.89 \mathrm{~b}$ & $3.96 \mathrm{~b}$ & $476 \mathrm{~b}$ \\
\hline \multicolumn{2}{|l|}{3.0 tonfed. $^{-1}$} & $0.79 \mathrm{c}$ & $3.76 \mathrm{c}$ & $451 \mathrm{c}$ & $0.79 \mathrm{c}$ & $3.74 \mathrm{c}$ & $449 \mathrm{c}$ \\
\hline \multicolumn{2}{|l|}{0.0 ton ed. $^{-1}$} & $0.71 d$ & $3.34 \mathrm{~d}$ & $401 d$ & $0.73 d$ & $3.51 \mathrm{~d}$ & $421 d$ \\
\hline
\end{tabular}

$* \mathrm{Ardb}=120 \mathrm{~kg}$

The interaction between irrigation regimes and bentonite conditioner was found to be significant in both seasons. The maximum seed yield 736 and $700 \mathrm{~kg}$ fed $^{-1}$ were recorded from treatment-received irrigation at 1.2 pan coefficient and received 6.0 ton $\mathrm{fed}^{-1}$ bentonite conditioner at first and second season, respectively. While the minimum seed yield 224 and $248 \mathrm{~kg} \mathrm{fed}^{-1}$ were recorded from treatment-received irrigation 0.8 pan coefficient and applied with zero ton $\mathrm{fed}^{-1}$ bentonite soil conditioner at first and second season, respectively.

Water use efficiency (WUE) of both lupine and sesame cropping system 
Water use efficiency (WUE) expressed as kg grain yield per one cubic meter of water consumed for both lupine and sesame crops as affected by 1.2, 1.0 and 0.8 pan and applying various rates of bentonite soil conditioner are presented in Table 10.

\section{Lupine}

With respect to lupine crop, results show that the effect of irrigation on the studied growth character was significant in both studied seasons. The values of water use efficiency could be increased by increasing crop yield and bentonite rates application as well as evapotranspiration being decreased. At first season the highest value of studied character was gained from 1.2 but it was from 1.0 pan coefficient irrigation treatment in second season followed by 0.8 pan treatment in both seasons. The difference between three irrigation treatments were significant at first season but the difference between 1.2 and 1.0 pan treatments was insignificant, whereas such value was significant at 0.8 pan coefficient in the second season. It can be concluded that moderate irrigation intervals are preferable for consuming water more efficiently. It is well known that plant roots extract water from greater depths than plants kept irrigated to optimum levels, thus water is used more efficiently. Similar results were found by El-Sherif and El-Hady (1986).

TABLE 10. Water use efficiency (WUE) as affected by different values of pan and bentonite soil conditioner application of both lupine and sesame crop rotation.

\begin{tabular}{|c|c|c|c|c|c|}
\hline \multirow{2}{*}{$\begin{array}{c}\text { Irrigation } \\
\text { Regime }\end{array}$} & \multirow{2}{*}{$\begin{array}{l}\text { Bent. Rates } \\
\text { ton fed. }\end{array}$} & \multicolumn{2}{|c|}{ Lupine } & \multicolumn{2}{|c|}{ Sesame } \\
\hline & & 2012- 2013 & 2013-2014 & 2013 & 2014 \\
\hline \multirow{4}{*}{1.2 pan coe. } & 6.0 & $0.610 \mathrm{ab}$ & $0.620 \mathrm{ab}$ & $0.234 \mathrm{a}$ & $0.215 \mathrm{a}$ \\
\hline & 4.5 & $0.553 \mathrm{bc}$ & $0.533 \mathrm{~cd}$ & $0.20 \mathrm{ab}$ & $0.181 \mathrm{ab}$ \\
\hline & 3.0 & $0.517 \mathrm{~cd}$ & $0.490 \mathrm{de}$ & $0.178 \mathrm{abc}$ & $0.166 \mathrm{ab}$ \\
\hline & 0.0 & $0.477 \mathrm{de}$ & $0.453 \mathrm{ef}$ & $0.163 \mathrm{~b}-\mathrm{e}$ & $0.151 \mathrm{bcd}$ \\
\hline \multicolumn{2}{|c|}{ Mean for irrigation } & 0539a & $0.524 \mathrm{a}$ & $0.194 \mathrm{a}$ & $0.178 \mathrm{a}$ \\
\hline \multirow{4}{*}{1.0 pan coe. } & 6.0 & $0.623 a$ & $0.667 \mathrm{a}$ & $0.232 \mathrm{a}$ & $0.216 \mathrm{a}$ \\
\hline & 4.5 & $0.557 \mathrm{bc}$ & $0.567 \mathrm{bc}$ & $0.189 \mathrm{abc}$ & $0.175 \mathrm{ab}$ \\
\hline & 3.0 & $0.500 \mathrm{~cd}$ & $0.503 \mathrm{de}$ & $0.160 \mathrm{bcd}$ & $0.160 \mathrm{abc}$ \\
\hline & 0.0 & $0.420 \mathrm{f}$ & $0.420 \mathrm{fg}$ & $0.138 \mathrm{c}-\mathrm{f}$ & $0.143 b-\mathrm{e}$ \\
\hline \multicolumn{2}{|c|}{ Mean for irrigation } & $0.525 \mathrm{a}$ & $0.539 \mathrm{a}$ & $0.179 \mathrm{a}$ & $0.173 a$ \\
\hline \multirow{4}{*}{0.8 pan coe. } & 6.0 & $0.420 \mathrm{ef}$ & $0.447 \mathrm{efg}$ & $0.117 d-f$ & $0.120 \mathrm{~b}-\mathrm{e}$ \\
\hline & 4.5 & $0.363 \mathrm{fg}$ & $0.393 \mathrm{gh}$ & $0.105 \mathrm{~d}-\mathrm{f}$ & $0.103 \mathrm{cde}$ \\
\hline & 3.0 & $0.343 \mathrm{~g}$ & $0.350 \mathrm{hi}$ & $0.098 \mathrm{ef}$ & $0.091 \mathrm{de}$ \\
\hline & 0.0 & $0.327 \mathrm{~g}$ & $0.320 \mathrm{i}$ & $0.082 \mathrm{f}$ & $0.086 \mathrm{e}$ \\
\hline \multicolumn{2}{|c|}{ Mean for irrigation } & $0.363 b$ & $0.378 b$ & $0.100 \mathrm{~b}$ & $0.100 \mathrm{~b}$ \\
\hline \multicolumn{6}{|c|}{ Mean for bent. conditioner } \\
\hline \multicolumn{2}{|l|}{6.0 ton/ fed. } & $0.551 \mathrm{a}$ & $0.578 \mathrm{a}$ & $0.194 \mathrm{a}$ & $0.184 \mathrm{a}$ \\
\hline \multicolumn{2}{|l|}{4.5 ton/ fed. } & $0.491 \mathrm{~b}$ & $0.498 \mathrm{~b}$ & $0.164 \mathrm{ab}$ & $0.153 \mathrm{ab}$ \\
\hline \multicolumn{2}{|l|}{3.0 ton/ fed. } & $0.453 \mathrm{c}$ & $0.448 \mathrm{c}$ & $0.145 \mathrm{bc}$ & $0.139 \mathrm{~b}$ \\
\hline \multicolumn{2}{|l|}{0.0 ton/ fed. } & $0.408 \mathrm{~d}$ & $0.398 \mathrm{~d}$ & $0.126 \mathrm{c}$ & $0.127 \mathrm{~b}$ \\
\hline
\end{tabular}

Water use efficiency by bentonite soil conditioner could be detected from data recorded in Table 10. Results indicated that the effect of bentonite conditioner on the studied character was significant in both seasons. Results clearly indicated that WUE increased from 0.408 to 0.551 in the first season and

Egypt. J. Soil. Sci. 56, No. 2 (2016) 
from 0.398 to 0.578 in second season. Obtained data may be due to increased rate of applied bentonite soil conditioner from zero to 6.0 ton $\mathrm{fed}^{-1}$ which increase soil water ability and decrease the water evaporation in such poor sandy soils (Hassan and Mahmoud, 2013).

The effect of interaction between irrigation treatments and bentonite soil conditioner rates on the studied character was significant in both seasons.

\section{Sesame}

Sesame water use efficiency (WUE) was influenced by irrigation intervals and rate of bentonite application. The obtained data are presented in Table 10. All variables significantly affected sesame water use efficiency in both seasons. The results showed that 1.2 pan coefficient treatment produced the maximum water use efficiency followed by the other treatments. The lowest WUE was obtained from the 0.8 pan coefficient and zero bentonite rates. The differences between irrigation treatments $(1.2,1.0$ and 0.8 pan) for WUE were significant in first season but such differences in second season between the irrigation treatments, i.e. 1.2 and 1.0 pan coefficients were insignificant as well as such relationship was significant between the 1.0 pan coefficient treatment and the 0.8 pan coefficient in second season. The previous result is in full agreement with those reported by Rafi et al. (2008).

Regarding to the effect of bentonite soil conditioner on WUE, the results show that the differences between bentonite rates were significant. The best response to bentonite conditioner on WUE is the application of 6.0 ton fed $^{-1}$. These results are in harmony with those obtained by Abou-Gabal et al. (1990).

The interaction between the irrigation regimes and the applied rates of bentonite was significant for both seasons.

\section{Total content of macronutrients for both lupine and sesame crops:}

Data in Tables 11\&12 showed the macronutrients total contents of lupine and sesame crops during two successive winter and summer seasons. The results reveal that high-frequency schedule (1.2 pan) gave the maximum grains or/ seeds and straw macronutrients total contents $(\mathrm{N}, \mathrm{P}$, and $\mathrm{K}$ ) followed by the other irrigation treatments. The irrigation treatments 0.8 pan coefficient recorded the lowest values for $\mathrm{N}, \mathrm{P}$ and $\mathrm{K}$ in grains or/ seeds and straw for both seasons. Similar results were obtained by Podsiadło and Kotlarz (2001) who mentioned that application of overhead irrigation and mineral fertilization on white Lupine cultivated on sandy soil resulted in increasing the yield of seeds. Furthermore, the efficiency of NPK increased on watered plots. Hassan and Mahmoud (2013) added that, due to low water retention of sandy soil, it needs frequent irrigations at short intervals. Understanding the sorption process in natural soil conditioner is necessary for effective utilization of these minerals as nutrient adsorbents and on the other side for controlling release of plant nutrients.

Regarding the application different rates of bentonite soil conditioner, results obtained generally showed that applied 6 ton $\mathrm{fed}^{-1}$ bentonite, compared to other treatments, were significantly higher for $\mathrm{N}, \mathrm{P}$ and $\mathrm{K}$ total content for straw and 
grains/or seeds of lupine and sesame plants, respectively. Furthermore, values of nutrients total content were more stimulated with application of the second and third of rates as compared to control (without bentonite) application. From this perspective, introducing clay-rich bentonite can improve the physical and chemical characteristics of sandy soil. This action will increase cation exchange capacity (Dejou, 1987) and improve soil structure leading to good water and nutrients retention and better soil ventilation (Raimund \& Dietmar, 1996 and Benkhalifa, 1997).

The statistical interaction analyses in Tables $11 \& 12$ showed that all applied treatments increased the total content of macronutrients over the control treatment; this trend was true for both straw and grains or seeds of lupine and sesame crops during two successive seasons. The high-frequency of irrigation (1.2 pan coefficient) and applied 6 ton fed $^{-1}$ gave the maximum grains/or seeds and straw $\mathrm{N}, \mathrm{P}$ and $\mathrm{K}$ total contents for lupine and sesame crops, respectively, followed by the other treatments. The lowest values of total content N, P and $\mathrm{K}$ were recorded under 0.8 pan coefficient irrigation treatment without applied bentonite. Obtained data agreed with Croker et al. (2004) and Noble et al. (2005), they mention that when applied bentonite to low CEC soils, it can bring about significant increases in the CEC simply as a consequence of their high net permanent negative charge. As a factor of increasing soil CEC, bentonite can also improve the retention and availability of nutrients, enhancing agricultural productivity and improving fertilizer use efficiency. A further benefit of bentonite is that it has the capacity to increase plant available water (PAW) as a function of increasing porosity (Suzuki, 2004 and Soda et al. 2006).

\section{Conclusion}

In Egypt, sandy soil has poor properties, i.e., low specific surface area, low water retention, low organic matter content, low fertility and high infiltration rate. These poor physical properties cause insufficient water use, especially in arid and semi arid regions. These adverse factors can be solved by several means; such as different natural conditioners (bentonite) and good irrigation management to improve poor soils. For both lupine and sesame cropping system, the highest actual evapotranspiration (ETa) was recorded under 1.2 accumulative pan evaporation treatment, whereas applied 6.0 ton $^{-1} \mathrm{~d}^{-1}$ bentonite recorded the lowest values of ETa in both seasons, as well as the highest value was obtained under zero ton $\mathrm{fed}^{-1}$ bentonite for the same respective crops. Also, under Ismailia condition it could be mentioned that pan evaporation and Hargreaves equations are more suitable used for scheduling irrigation for lupine and sesame crops compared with the other equations. Yield of both lupine and sesame (straw and grain/or seeds) along with total content of macronutrients ( $\mathrm{N}, \mathrm{P}$ and $\mathrm{K}$ ) increased significantly under the irrigation treatment of 1.2 pan in presence of 6.0 ton fed ${ }^{-1}$ bentonite mineral soil conditioner as compared to other treatments. 
EFFECT OF IRRIGATION REGIME AND NATURAL SOIL CONDITIONER 345

TABLE 11. Nutrients total contents of lupine crop as affected by irrigation scheduling using pan and different rates of bentonite soil conditioner during two winter seasons.

\begin{tabular}{|c|c|c|c|c|c|c|c|c|c|c|c|c|c|}
\hline \multirow{3}{*}{$\begin{array}{c}\text { Irrg. } \\
\text { Regi } \\
\text { me }\end{array}$} & \multirow{3}{*}{$\mid \begin{array}{c}\text { Bent. Rates } \\
\text { ton fed }^{-1}\end{array}$} & \multicolumn{6}{|c|}{ Season 2012-2013 } & \multicolumn{6}{|c|}{ Season 2013-2014 } \\
\hline & & \multicolumn{3}{|c|}{$\begin{array}{l}\text { Straw total content } \\
\text { Kg fed }^{-1}\end{array}$} & \multicolumn{3}{|c|}{$\begin{array}{c}\text { Grains total content } \\
\mathrm{Kg} \mathrm{fed}^{-1}\end{array}$} & \multicolumn{3}{|c|}{$\begin{array}{c}\text { Straw total content } \\
\mathrm{Kg} \mathrm{fed}^{-1}\end{array}$} & \multicolumn{3}{|c|}{$\begin{array}{c}\text { Grains total content } \\
\mathrm{Kg} \mathrm{fed}^{-1}\end{array}$} \\
\hline & & $\mathbf{N}$ & $\mathbf{P}$ & $\mathbf{K}$ & $\mathbf{N}$ & $\mathbf{P}$ & $\mathbf{K}$ & $\mathbf{N}$ & $\mathbf{P}$ & $\mathbf{K}$ & $\mathbf{N}$ & $\mathbf{P}$ & $\mathbf{K}$ \\
\hline \multirow{4}{*}{$\begin{array}{l}1.2 \\
\text { pan. }\end{array}$} & 6.0 & 47.61a & $8.41 \mathrm{a}$ & $7.08 \mathrm{a}$ & $28.02 \mathrm{a}$ & $6.89 \mathrm{a}$ & $5.74 \mathrm{a}$ & $52.06 \mathrm{a}$ & $9.22 \mathrm{a}$ & $7.72 \mathrm{a}$ & $28.98 \mathrm{a}$ & $7.09 \mathrm{a}$ & $5.93 \mathrm{a}$ \\
\hline & 4.5 & $38.10 \mathrm{~b}$ & $6.44 \mathrm{~b}$ & $4.71 \mathrm{~b}$ & $26.09 \mathrm{~b}$ & $5.71 \mathrm{ab}$ & $5.33 \mathrm{a}$ & $49.27 \mathrm{a}$ & $8.36 \mathrm{a}$ & $6.10 \mathrm{~b}$ & $25.80 \mathrm{~b}$ & $5.63 \mathrm{ab}$ & $5.27 \mathrm{~b}$ \\
\hline & 3.0 & $32.77 \mathrm{c}$ & $5.59 \mathrm{bc}$ & $3.85 \mathrm{~b}$ & $23.10 \mathrm{c}$ & $5.26 \mathrm{ab}$ & $4.76 \mathrm{~b}$ & $30.47 \mathrm{~b}$ & $5.21 \mathrm{~b}$ & $3.58 \mathrm{c}$ & $23.01 \mathrm{c}$ & $5.24 \mathrm{ab}$ & $4.74 \mathrm{c}$ \\
\hline & 0.0 & $29.33 \mathrm{c}$ & $4.72 \mathrm{~cd}$ & $3.28 \mathrm{bcd}$ & $20.09 \mathrm{~d}$ & $4.66 \mathrm{bc}$ & $4.32 \mathrm{bc}$ & $29.58 \mathrm{~b}$ & $4.78 \mathrm{bc}$ & $3.30 \mathrm{~cd}$ & $20.38 \mathrm{~d}$ & $4.72 \mathrm{bc}$ & $4.38 \mathrm{~cd}$ \\
\hline \multicolumn{2}{|c|}{ Mean for irrigation } & $36.95 \mathrm{a}$ & $6.29 \mathrm{a}$ & $4.73 \mathrm{a}$ & $24.33 \mathrm{a}$ & $5.63 \mathrm{a}$ & $5.04 \mathrm{a}$ & $40.34 \mathrm{a}$ & $6.89 \mathrm{a}$ & $5.18 \mathrm{a}$ & $24.5 \mathrm{a}$ & $5.67 \mathrm{a}$ & $5.08 \mathrm{a}$ \\
\hline \multirow{4}{*}{$\begin{array}{l}1.0 \\
\text { pan. }\end{array}$} & 6.0 & $21.77 \mathrm{~d}$ & $4.59 \mathrm{~cd}$ & $3.72 \mathrm{bc}$ & $21.79 \mathrm{c}$ & $4.49 \mathrm{bcd}$ & $4.52 \mathrm{bc}$ & $22.83 \mathrm{c}$ & $4.84 \mathrm{bc}$ & $3.89 \mathrm{c}$ & $22.85 \mathrm{c}$ & $4.69 \mathrm{bc}$ & $4.74 \mathrm{c}$ \\
\hline & 4.5 & $18.59 \mathrm{de}$ & $3.81 \mathrm{de}$ & $3.12 \mathrm{bcd}$ & $19.10 \mathrm{~d}$ & $3.93 \mathrm{~b}-\mathrm{e}$ & $4.18 \mathrm{c}$ & $19.16 \mathrm{~cd}$ & $3.68 \mathrm{bcd}$ & $2.99 \mathrm{c}-\mathrm{e}$ & $19.27 \mathrm{~d}$ & $3.96 \mathrm{~b}-\mathrm{d}$ & $4.22 \mathrm{~d}$ \\
\hline & 3.0 & 17.44de & $2.79 \mathrm{ef}$ & $2.18 \mathrm{ce}$ & $17.37 \mathrm{e}$ & $3.13 \mathrm{c}-\mathrm{e}$ & $3.68 \mathrm{~d}$ & $18.91 \mathrm{~cd}$ & $3.07 \mathrm{c}-\mathrm{e}$ & $2.39 \mathrm{c}-\mathrm{f}$ & $17.27 \mathrm{e}$ & $3.11 \mathrm{~cd}$ & $3.66 \mathrm{e}$ \\
\hline & 0.0 & $16.27 \mathrm{~d}-\mathrm{f}$ & $2.55 \mathrm{ef}$ & $1.93 \mathrm{de}$ & $14.28 \mathrm{f}$ & $2.65 \mathrm{c}-\mathrm{e}$ & $3.23 \mathrm{de}$ & $17.88 \mathrm{~cd}$ & 2.91de & $2.23 \mathrm{c}-\mathrm{f}$ & $14.50 \mathrm{f}$ & $2.69 \mathrm{~cd}$ & $3.27 \mathrm{e}$ \\
\hline \multicolumn{2}{|c|}{ Mean for irrigation } & $18.52 \mathrm{~b}$ & $3.44 \mathrm{~b}$ & $2.74 \mathrm{~b}$ & $18.13 b$ & $3.55 \mathrm{~b}$ & $3.90 \mathrm{~b}$ & $19.69 \mathrm{~b}$ & $3.63 \mathrm{~b}$ & $2.88 \mathrm{~b}$ & $18.47 \mathrm{~b}$ & $3.62 \mathrm{~b}$ & $3.98 \mathrm{~b}$ \\
\hline \multirow{4}{*}{$\begin{array}{l}0.8 \\
\text { Pan. }\end{array}$} & 6.0 & $13.18 \mathrm{e}-\mathrm{g}$ & $2.65 \mathrm{ef}$ & $1.86 \mathrm{de}$ & $12.16 \mathrm{~g}$ & $3.02 \mathrm{c}-\mathrm{e}$ & $2.74 \mathrm{ef}$ & $13.28 \mathrm{de}$ & $2.67 \mathrm{de}$ & $1.88 \mathrm{df}$ & $12.09 \mathrm{~g}$ & $3.00 \mathrm{~cd}$ & $2.72 \mathrm{f}$ \\
\hline & 4.5 & $11.04 \mathrm{fg}$ & $1.81 \mathrm{f}$ & $1.42 \mathrm{e}$ & $10.73 \mathrm{~h}$ & $2.45 \mathrm{de}$ & $2.28 \mathrm{fg}$ & $11.57 \mathrm{e}$ & $1.91 \mathrm{de}$ & $1.49 \mathrm{ef}$ & $11.06 \mathrm{gh}$ & $2.54 \mathrm{~d}$ & $2.35 \mathrm{fg}$ \\
\hline & 3.0 & $9.42 \mathrm{~g}$ & $1.58 \mathrm{f}$ & $1.24 \mathrm{e}$ & $10.26 \mathrm{~h}$ & $2.39 \mathrm{e}$ & $2.13 \mathrm{~g}$ & $9.54 \mathrm{e}$ & $1.60 \mathrm{e}$ & $1.25 \mathrm{f}$ & $10.09 \mathrm{hi}$ & $2.36 \mathrm{~d}$ & $2.09 \mathrm{~g}$ \\
\hline & 0.0 & $8.69 \mathrm{~g}$ & $1.50 \mathrm{f}$ & $1.18 \mathrm{e}$ & $9.96 \mathrm{~h}$ & $1.97 \mathrm{e}$ & $2.00 \mathrm{~g}$ & $8.56 \mathrm{e}$ & $1.49 \mathrm{e}$ & $1.16 \mathrm{f}$ & $9.46 \mathrm{i}$ & $1.88 \mathrm{~d}$ & $1.90 \mathrm{~g}$ \\
\hline \multicolumn{2}{|c|}{ Mean for irrigation } & $10.58 \mathrm{c}$ & $1.88 \mathrm{~b}$ & $1.43 \mathrm{c}$ & $10.78 \mathrm{c}$ & $2.46 \mathrm{~b}$ & $2.29 \mathrm{c}$ & $10.74 \mathrm{c}$ & $1.92 \mathrm{~b}$ & $1.44 \mathrm{c}$ & $10.67 \mathrm{c}$ & $2.45 \mathrm{~b}$ & $2.27 \mathrm{c}$ \\
\hline \multicolumn{14}{|c|}{ Mean for bent. Conditioner } \\
\hline \multicolumn{2}{|c|}{6.0 ton fed. $^{-1}$} & $27.52 \mathrm{a}$ & $5.22 \mathrm{a}$ & $4.22 \mathrm{a}$ & $20.65 \mathrm{a}$ & $4.80 \mathrm{a}$ & $4.33 \mathrm{a}$ & $29.39 \mathrm{a}$ & $5.58 \mathrm{a}$ & $4.49 \mathrm{a}$ & 21.31a & $4.93 \mathrm{a}$ & $4.46 \mathrm{a}$ \\
\hline \multicolumn{2}{|c|}{4.5 ton fed. ${ }^{-1}$} & $22.58 \mathrm{~b}$ & $4.02 \mathrm{~b}$ & $3.09 \mathrm{~b}$ & $18.64 \mathrm{~b}$ & $4.03 \mathrm{ab}$ & $3.92 \mathrm{~b}$ & $26.24 \mathrm{a}$ & $4.65 \mathrm{a}$ & $3.53 \mathrm{~b}$ & $18.71 \mathrm{~b}$ & $4.04 \mathrm{ab}$ & $3.95 \mathrm{~b}$ \\
\hline \multicolumn{2}{|c|}{3.0 ton fed. ${ }^{-1}$} & $19.88 \mathrm{bc}$ & $3.32 \mathrm{bc}$ & $2.42 \mathrm{bc}$ & $16.91 \mathrm{c}$ & $3.60 \mathrm{~b}$ & $3.52 \mathrm{c}$ & $19.72 \mathrm{~b}$ & $3.29 \mathrm{~b}$ & $2.41 \mathrm{c}$ & $16.79 \mathrm{c}$ & $3.57 \mathrm{~b}$ & $3.49 \mathrm{c}$ \\
\hline \multicolumn{2}{|c|}{0.0 tonfed. $^{-1}$} & $18.09 \mathrm{c}$ & $2.92 \mathrm{c}$ & $2.13 \mathrm{c}$ & $14.78 \mathrm{~d}$ & $3.09 \mathrm{~b}$ & $3.18 \mathrm{~d}$ & $19.02 \mathrm{~b}$ & $3.06 \mathrm{~b}$ & $2.23 \mathrm{c}$ & $14.78 \mathrm{~d}$ & $3.10 \mathrm{~b}$ & $3.19 \mathrm{~d}$ \\
\hline
\end{tabular}


TABLE 12. Nutrients total contents of sesame crop as affected by irrigation scheduling using pan and different rates of bentonite soil conditioner during two summer seasons.

\begin{tabular}{|c|c|c|c|c|c|c|c|c|c|c|c|c|c|}
\hline \multirow{3}{*}{$\begin{array}{c}\text { Irrg. } \\
\text { Regime }\end{array}$} & \multirow{3}{*}{$\begin{array}{c}\text { Bent. Rates } \\
\text { ton fed. }{ }^{-1}\end{array}$} & \multicolumn{6}{|c|}{ Season 2013} & \multicolumn{6}{|c|}{ Season 2014} \\
\hline & & \multicolumn{3}{|c|}{$\begin{array}{l}\text { Straw total content } \\
\text { Kg fed- }^{-1}\end{array}$} & \multicolumn{3}{|c|}{$\begin{array}{l}\text { Grains total content } \\
\text { Kg fed- }^{1}\end{array}$} & \multicolumn{3}{|c|}{$\begin{array}{l}\text { Straw total content } \\
\text { Kg fed- }^{1}\end{array}$} & \multicolumn{3}{|c|}{$\begin{array}{c}\text { Grains total content } \\
\text { Kg fed- }{ }^{1}\end{array}$} \\
\hline & & $\mathbf{N}$ & $\mathbf{P}$ & $\mathbf{K}$ & $\mathbf{N}$ & $\mathbf{P}$ & $\mathbf{K}$ & $\mathbf{N}$ & $\mathbf{P}$ & $\mathbf{K}$ & $\mathbf{N}$ & $\mathbf{P}$ & $\mathbf{K}$ \\
\hline \multirow{4}{*}{1.2 pan. } & 6.0 & $20.4 \mathrm{a}$ & $4.47 \mathrm{ab}$ & $10.2 \mathrm{a}$ & $28.0 \mathrm{a}$ & $6.89 \mathrm{a}$ & $5.74 \mathrm{a}$ & $19.4 \mathrm{a}$ & $4.18 \mathrm{ab}$ & $9.70 \mathrm{a}$ & $28.9 \mathrm{a}$ & $7.09 \mathrm{a}$ & $5.93 \mathrm{a}$ \\
\hline & 4.5 & $18.1 \mathrm{~b}$ & $4.26 \mathrm{ab}$ & $8.64 \mathrm{ab}$ & $26.1 \mathrm{~b}$ & $5.71 \mathrm{ab}$ & $5.33 \mathrm{a}$ & $17.1 \mathrm{~b}$ & $4.02 \mathrm{ab}$ & $8.16 \mathrm{~b}$ & $25.8 \mathrm{~b}$ & $5.63 \mathrm{ab}$ & $5.27 \mathrm{~b}$ \\
\hline & 3.0 & $17.1 \mathrm{~b}$ & $3.84 \mathrm{abc}$ & $8.13 \mathrm{ab}$ & $23.1 \mathrm{c}$ & $5.26 \mathrm{ab}$ & $4.76 \mathrm{~b}$ & $16.8 \mathrm{~b}$ & $3.80 \mathrm{ab}$ & $7.96 \mathrm{~b}$ & $23.0 \mathrm{c}$ & $5.23 \mathrm{ab}$ & $4.74 \mathrm{c}$ \\
\hline & 0.0 & $15.7 \mathrm{c}$ & $3.80 \mathrm{abc}$ & $7.67 \mathrm{abc}$ & $20.1 \mathrm{~d}$ & $4.66 \mathrm{bc}$ & $4.32 \mathrm{bc}$ & $15.7 \mathrm{bc}$ & $3.79 \mathrm{ab}$ & $7.67 \mathrm{~b}$ & $20.4 \mathrm{~d}$ & $4.71 b c$ & $4.38 \mathrm{~cd}$ \\
\hline \multicolumn{2}{|c|}{ Mean for irrigation } & $17.8 \mathrm{a}$ & $4.09 \mathrm{a}$ & $8.67 \mathrm{a}$ & $24.3 \mathrm{a}$ & $5.62 \mathrm{a}$ & $5.04 \mathrm{a}$ & $17.2 \mathrm{a}$ & $3.95 \mathrm{a}$ & $8.37 \mathrm{a}$ & $8.37 \mathrm{a}$ & $5.67 \mathrm{a}$ & $5.08 \mathrm{a}$ \\
\hline \multirow{4}{*}{$1.0 \mathrm{pan}$} & 6.0 & $18.0 \mathrm{~b}$ & $5.30 \mathrm{a}$ & $9.91 \mathrm{a}$ & $21.8 \mathrm{c}$ & $4.49 \mathrm{bcd}$ & $4.52 \mathrm{bc}$ & $17.1 \mathrm{~b}$ & $5.02 \mathrm{a}$ & $9.36 \mathrm{a}$ & $22.9 \mathrm{c}$ & $4.69 \mathrm{bc}$ & $4.74 \mathrm{c}$ \\
\hline & 4.5 & $15.6 \mathrm{c}$ & $4.13 \mathrm{a}-\mathrm{d}$ & 7.77abc & $19.1 \mathrm{~d}$ & 3.93bcde & $4.18 \mathrm{c}$ & $14.9 \mathrm{~cd}$ & $4.30 \mathrm{ab}$ & $7.45 \mathrm{~b}$ & $19.3 \mathrm{~d}$ & $3.96 \mathrm{bcd}$ & $4.22 \mathrm{~d}$ \\
\hline & 3.0 & $13.3 \mathrm{~d}$ & $3.47 \mathrm{ab}$ & $6.31 \mathrm{bc}$ & $17.4 \mathrm{e}$ & $3.13 \mathrm{cde}$ & $3.68 \mathrm{~d}$ & $13.8 \mathrm{~d}$ & $3.31 \mathrm{abc}$ & $6.57 \mathrm{c}$ & $17.3 \mathrm{e}$ & $3.11 \mathrm{~cd}$ & $3.66 \mathrm{e}$ \\
\hline & 0.0 & $12.7 \mathrm{~d}$ & $2.76 \mathrm{~b}-\mathrm{d}$ & $5.22 \mathrm{~cd}$ & $14.3 \mathrm{f}$ & $2.65 \mathrm{cde}$ & 3.23de & $13.8 \mathrm{~d}$ & 2.99abc & $5.64 \mathrm{~d}$ & $14.5 \mathrm{f}$ & $2.69 \mathrm{~cd}$ & $3.27 \mathrm{e}$ \\
\hline \multicolumn{2}{|c|}{ Mean for irrigation } & $14.9 \mathrm{~b}$ & $3.92 \mathrm{~b}$ & $7.31 \mathrm{~b}$ & $18.1 \mathrm{~b}$ & $3.55 \mathrm{~b}$ & $3.90 \mathrm{a}$ & $14.9 \mathrm{~b}$ & $3.90 \mathrm{~b}$ & $7.25 b$ & $7.25 \mathrm{~b}$ & $3.62 \mathrm{~b}$ & $3.98 b$ \\
\hline \multirow{4}{*}{ 0.8 Pan. } & 6.0 & $8.30 \mathrm{e}$ & $2.68 \mathrm{~b}-\mathrm{d}$ & $3.57 \mathrm{~d}$ & $12.2 \mathrm{~g}$ & $3.02 \mathrm{cde}$ & $2.74 \mathrm{ef}$ & $9.56 \mathrm{e}$ & 3.19abc & $4.09 \mathrm{e}$ & $12.1 \mathrm{~g}$ & $3.00 \mathrm{~cd}$ & $2.72 \mathrm{f}$ \\
\hline & 4.5 & $7.99 \mathrm{ef}$ & $2.53 b-d$ & $3.53 \mathrm{~d}$ & $10.7 \mathrm{~h}$ & $2.45 \mathrm{de}$ & $2.28 \mathrm{fg}$ & $8.01 \mathrm{ef}$ & $2.52 \mathrm{bc}$ & $3.53 \mathrm{ef}$ & $11.1 \mathrm{gh}$ & $2.54 \mathrm{~d}$ & $2.35 \mathrm{fg}$ \\
\hline & 3.0 & $7.32 \mathrm{ef}$ & $1.63 \mathrm{~d}$ & $3.36 \mathrm{~d}$ & $10.3 \mathrm{~h}$ & $2.39 \mathrm{e}$ & $2.13 \mathrm{~g}$ & $6.98 \mathrm{f}$ & $2.16 \mathrm{c}$ & $3.20 \mathrm{f}$ & $10.1 \mathrm{hi}$ & $2.36 \mathrm{~d}$ & $2.09 \mathrm{~g}$ \\
\hline & 0.0 & 7.09f & $1.96 \mathrm{~cd}$ & $2.68 \mathrm{~d}$ & $9.96 \mathrm{~h}$ & $1.97 \mathrm{e}$ & $2.00 \mathrm{~g}$ & $7.84 \mathrm{f}$ & $1.57 \mathrm{bc}$ & $2.97 \mathrm{f}$ & $9.5 \mathrm{i}$ & $1.88 \mathrm{~d}$ & $1.90 \mathrm{~g}$ \\
\hline \multicolumn{2}{|c|}{ Mean for irrigation } & $7.68 \mathrm{c}$ & $2.20 \mathrm{c}$ & $3.29 \mathrm{c}$ & $10.8 \mathrm{c}$ & $2.46 \mathrm{~b}$ & $2.29 \mathrm{~b}$ & $8.10 \mathrm{c}$ & $2.36 \mathrm{c}$ & $3.45 \mathrm{c}$ & $3.44 \mathrm{c}$ & $2.45 \mathrm{~b}$ & $2.27 \mathrm{c}$ \\
\hline \multicolumn{14}{|c|}{ Mean for bent. Conditioner } \\
\hline \multicolumn{2}{|c|}{6.0 ton fed $^{-1}$. } & $15.6 \mathrm{a}$ & $4.15 \mathrm{a}$ & $7.90 \mathrm{a}$ & $20.7 \mathrm{a}$ & $4.80 \mathrm{a}$ & $4.33 \mathrm{a}$ & $15.3 \mathrm{a}$ & $4.13 \mathrm{a}$ & $7.72 \mathrm{a}$ & $21.3 \mathrm{a}$ & $4.93 \mathrm{a}$ & $4.46 \mathrm{a}$ \\
\hline \multicolumn{2}{|c|}{4.5 ton fed. ${ }^{-1}$} & $13.9 \mathrm{~b}$ & $3.42 \mathrm{ab}$ & $6.65 \mathrm{ab}$ & $18.6 \mathrm{~b}$ & $4.03 \mathrm{ab}$ & $3.93 \mathrm{~b}$ & $13.4 \mathrm{~b}$ & $3.29 \mathrm{ab}$ & $6.38 \mathrm{ab}$ & $18.7 \mathrm{~b}$ & $4.04 \mathrm{ab}$ & $3.95 \mathrm{~b}$ \\
\hline \multicolumn{2}{|c|}{3.0 tonfed. $^{-1}$} & $12.6 \mathrm{c}$ & $3.20 \mathrm{ab}$ & $5.93 b$ & $16.9 \mathrm{c}$ & $3.60 \mathrm{~b}$ & $3.52 \mathrm{c}$ & $12.5 \mathrm{c}$ & $3.22 \mathrm{ab}$ & $5.91 \mathrm{~b}$ & $16.8 \mathrm{c}$ & $3.57 \mathrm{~b}$ & $3.49 \mathrm{c}$ \\
\hline \multicolumn{2}{|c|}{0.0 ton fed. ${ }^{-1}$} & $11.8 \mathrm{~d}$ & $2.84 \mathrm{~b}$ & $5.19 \mathrm{~b}$ & $14.8 \mathrm{~d}$ & $3.09 \mathrm{~b}$ & $3.18 \mathrm{~d}$ & $12.4 \mathrm{c}$ & $2.98 \mathrm{~b}$ & $5.42 \mathrm{~b}$ & $14.8 \mathrm{~d}$ & $3.09 \mathrm{~b}$ & $3.19 \mathrm{~d}$ \\
\hline
\end{tabular}

Acknowledgement: The authors wish to express their sincere gratitude and appreciation to the Development of Soil Conditioners Project, Dept. of Physics and Chemistry of Soil, Soils, Water and Environ. Res. Inst., Agric. Res. Center 
(ARC), Giza, Egypt, for introducing all facilities needed to accomplish this study.

\section{References}

Abd El-Hady, M. and Eldardiry, E. I. (2012) Sustainable reclamation of newly and natural zeolite on sandy soil properties and productivity of some crops. Top Class Jour. of Agric. Res., 1, 22-28.

Abou-Gabal, A., Abdel-Sabour, M.F., Mohamed, F.A. and Ragab, M.A. (1990) Feasibility of sandy soil reclamation using local tafla as soil conditioner. Annals Agric. Sci. Cairo, 34, 1003-1015.

Afifi, M.Y. (1986) Use of clay deposites in improving the physical properties of sandy soils. Jour. King Saud Univ. Agric. Sci. 8, 255-265.

Allen, R.G., Pereira, L.S., Raes, L. and Smith, M. (1998) Crop evapotranspiration Guideline for computing crop water requirements. Irrg. Drain paper No 56. Food and Agriclture Organization of the United Nations, Rome, Italy.

Al-Omran, A.M., Falatah, A.M., Sheta, A.S. and Al-Harbi, A.R. (2004) Clay deposits for water management of sandy soils. Arid Land Res. Manage. 18,171-184.

Benkhalifa, M. (1997) Influence of bentonite on the physical, hydric and mechanical properties of a sandy soil of the plateau of Mostaganem, Ph.D. Thesis, Algiers.

Cottenie, A., Verloo, M., Kiekens, L., Velghe, G. and Amertynck, R. (1982) Chemical Analysis of Plants and Soils. Laboratory of Analytical and Agro chemistry, State University, Ghent, Belgium. 50-70.

Croker, J., Poss, R., Hartmann, C. and Bhuthorndharaj, S. (2004) Effects of recycled, bentonite addition on soil properties, plant growth and nutrient uptake in a tropical sandy soil. Plant and Soil, 267, 155-163.

Cuenca, R.H. and Amegee, K.Y. (1987) Analysis of evapotranspiration as a regionalized variable. Advances Irrigation.vol .4.

Dejou, J. (1987) The specific surface of clay, its measurement, relationship with the CEC and its agronomic importance, in: Proceeding Symposium AFES, pp. 72-83.

Dmowski, Z. (1986) Influence of precipitation deficit or excess and irrigation on yielding of papilionaceous plants in 1952-76 in Poland. In: AGRIS since: 1987.

Doorenbos, J. and Kassam, A.H. (1979) Yield response to water. Irrg. Drain paper No 33. Food and Agriclture Organization of the United Nations, Rome, Italy.

Doorenbos, J. and Pruitt, W.O. (1977) Yield response to water. Irrg. Drain paper No 24. Food and Agriclture Organization of the United Nations, Rome, Italy.

El-Fahal, E.M. (2007) Effect of Irrigation interval, intra-row spacing and sowing date on seed yield and yield components of local lupine (lupinus term is forks.) in the Nile River State in Northern Sudan. 
El-Sherif, A.F. and El-Hady, O.A. (1986) The possibility of using Egyptian bentonite deposits for sandy soils reclamation and planting. Egypt. J. Soil. Sci. (Special Issue) 171.

Ferguson, J. (2015) Soil Amendments - Bentonite. www.natureswayresources.com.

Hargreave, G. H. and Samani, Z. A. (1985) Estimating potential evapotranspiration J. Irrig. and Drainage, ASCE, 108(3).

Hassan, A.Z.A. and Mahmoud, A.M. (2013) The combined effect of bentonite and natural zeolite on sandy soil properties and productivity of some crops. Top Class Journal of Agric., 1, 22-28.

Israelson, O.W. and Hansen, V.E. (1962) Irrigation Principles and Practices, John Wily and Sons Inc. New York.

Jansen, P. C. M. (2006) Lupinus albus (L.) Record from Protabase. Brink, M. \& Belay, G. (Editors). PROTA (Plant Resources of Tropical Africa / Ressources végétales de l'Afrique tropicale), Wageningen, Netherlands.

Jouyban, Z. and Moosavi, S.G. (2011) Study of effects of different levels of irrigation interval, nitrogen and superabsorbent on seed yield and morphological traits of Sesame. Australian Jour. of Basic and Appl. Sci., 5, 1317-1323.

Matloub, M.A. (1998) Effect of some soil conditioners on soil modulus of rupture, hydrophysical properties and plant growth at different soil moisture levels. Egypt. J. Applied Sci., 13, 350-365.

Nadeem, A., Kashani, S., Ahmed, N., Buriro, M., Saeed, Z., Mohammad, F. and Ahmed, S. (2015) Growth and yield of sesame (Sesamum indicum L.) under the influence of planting geometry and irrigation regimes. Amer. Jour.Plant Sci., 6, 980-986.

Noble, A.D., Ruaysoongnern, S., Berthelsen, S., Penning, de Vries, F.W.T. and Giordano, M. (2005) Enhancing the agronomic productivity of degraded soils through clay-based interventions. The International Jour. of Agric. Sustainability 3,102-113.

Noble, A.D, Nath, S. and Srivastave, R.J. (2001) Changes in the surface charge characteristics of degraded soils in the wet tropics through the addition of beneficiated bentonite. Aust. Jour. of Soil Res. 39, 991-1001.

Page, A.L., Miller, R.H. and Keeney, D.R. (1982) "Methods of Soil Analysis". Part II. Amer. Soc. Agron., Madison, Wisconsin, USA.

Podsiadło, C. and Kotlarz, A. (2001) Influence of irrigation and mineral fertilization on the yield and nutritional value of white lupine seeds. Inzynieria Rolnicza, 13, 370-376.

Rafi, M. R., Dehqani, A. and Shakeri, F.O. (2008) Study the effects of irrigation frequency on yield and yield components of sesame in Behbahan region. http://agrisis.areo.ir/HomePage.aspx?TabID=15589\&Site=agrisis.areo \&Lang=en-US

Egypt. J. Soil. Sci. 56, No. 2 (2016) 
Raimund, S. and Dietmar, S. (1996) Properties of soils under different types of management developed in a sandy substrate covering boulder clay at Mecklenburg (north eastern Germany), in: Sci. of Soils.

Sarhadi, J. and Sharif, M. (2014) The Effect of deficit irrigation on sesame growth, yield and yield components in drought conditions on base of sustainable agriculture. Intl J. Farm \& Alli Sci., 3, 1061-1064.

Schwen, A. and Loiskandl, W. (2010) Assessment of Soil-Amendment Mixtures for Subsurface Drip Irrigation Systems. $19^{\text {th }}$ World Congress of Soil Science, Soil Solutions for a Changing World 1-6 August 2010, Brisbane, Australia. Published on DVD.

Sendecor, G.W. and Cochran, W.G • (1967) Statistical Methods. Oxford and I.B.H. publishing.$^{\text {th }}$ ed.p.p.299-310.

Sepaskhah, A.R. and Andam, M . (2001) Crop coefficient of sesame in semi-arid region of I.R. Iran. Agric. Water Mange. 49, 51-63.

Shakoor, 1.A., Arshad, M., Tariq, A. and Ahmad, I. (2012) Evaluating the role of bentonite amendment in controlling infiltration and improve root zone water distribution in coarse soil. Pak. J. Agri. Sci., 49, 375-380.

Soda, W., Noble, A.D., Suzuki, S., Simmons, R., Sindhusen, L. and Bhuthorndharaj, S. (2006) Cocomposting of acid waste bentonites and their effects on soil properties and crop biomass. Jour. of Environ. Quality, 35, 2293-2301.

Stanhill, G. (2002) Is the class A evapotranspiration pan still the most practical and accurate meteorological method for determining irrigation water requirements? Agriculture and Forest Meterology, 112, 233-236.

Suzuki, S. (2004) Verification of freezing point depression method for measuring matric potential of soil water. Soil Sci. and Plant Nutr. 50, 1277-1280.

Toan, D. P., Thuy-Duong, T.N.A., Carlsson, S. and Bui, T.M. (2010) Morphological evaluation of sesame (Sesamum indicum L.) varieties from different origins. Australian Jour. of Crop Sci., 4, 498-504.

Ucan, K., Killi, F., Gencoglan, C. and Merdun, H. (2007) Effect of irrigation frequency and amount on water use efficiency and yield of sesame (Sesamum indicum L.) under field conditions. Field Crops Res. 101, 249-258.

Vites, F.G. (1965) Increasing water use efficiency by soil management. Amer. Sco. Agron., Madison,Wisc. P 259-274.

(Received: 15/4/2016;

accepted: 2/8/2016) 


\section{تأثير نظام الري ومحسنات التربة الطبيعية على إنتاجية المحاصيل في التربة الرملية

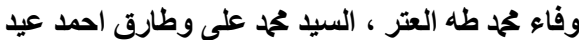 \\ معهد بحوث الار اضى وفئ والمياه و البيئة مركز البحوث احمث الزر اعية ـ جيزة - مصر.
}

اجريت تجربة حقلية فى اراضى رملية فى المزرعة البحثية- بمحطة البحوث

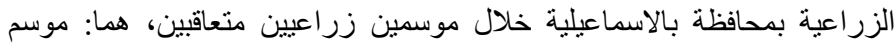

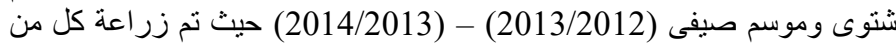

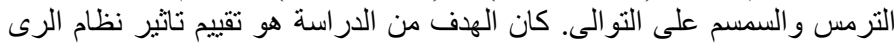

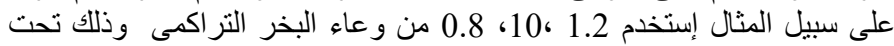

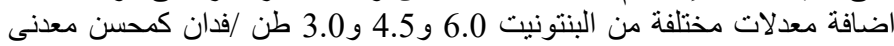

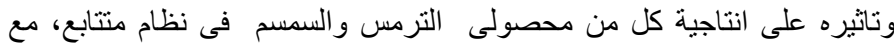

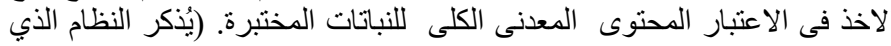
إستخدم في الري كما يُنكر العمق الذئي تم فيه خلط معدن الطين).

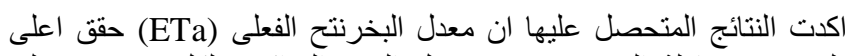

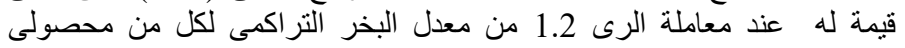

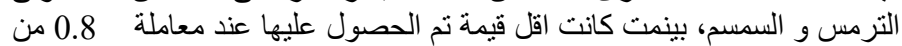

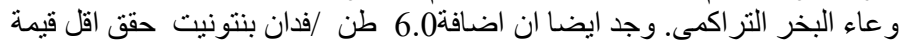

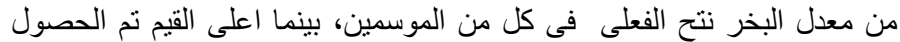
عليها عند عدم اضافة بنتونيت لكل من المحصول كن الين المتعاقبين.

بالاضافة الى ذلك فانه من المناسب وتحت ظروف محافظة الاسماعيلية ان تطبق معادلات الرى الى ن

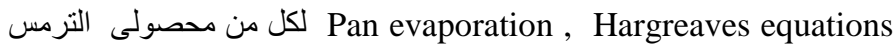
و السمسم بالمقارنة مع المعادلات الاخرى المختبرة.

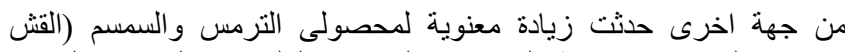

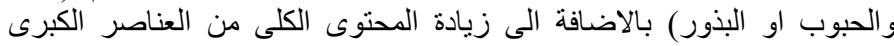

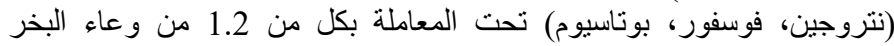

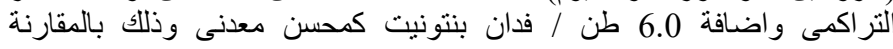

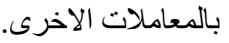

